LBL-37408

UC-414

Preprint

Submitted to Journal of Vacuum Science and Technology A

\title{
Ion Desorption Stability in Superconducting High Energy Physics Proton Colliders
}

W.C. Turner

May 1995 


\section{DISCLAIMER}

This document was prepared as an account of work sponsored by the United States Government. While this document is believed to contain correct information, neither the United States Government nor any agency thereof, nor The Regents of the University of California, nor any of their employees, makes any warranty, express or implied, or assumes any legal responsibility for the accuracy, completeness, or usefulness of any information, apparatus, product, or process disclosed, or represents that its use would not infringe privately owned rights. Reference herein to any specific commercial product, process, or service by its trade name, trademark, manufacturer, or otherwise, does not necessarily constitute or imply its endorsement, recommendation, or favoring by the United States Government or any agency thereof, or The Regents of the University of California. The views and opinions of authors expressed herein do not necessarily state or reflect those of the United States Government or any agency thereof, or The Regents of the University of California.

Lawrence Berkeley Laboratory is an equal opportunity employer. 


\title{
Ion Desorption Stability in Superconducting High Energy Physics Proton Colliders*
}

\author{
William C. Turner \\ Lawrence Berkeley Laboratory \\ University of California \\ Berkeley, California
}

29 May 1995

* This work was supported by the Director, Office of Energy Research, Office of High Energy and Nuclear Physics, High Energy Physics Division, U.S. Department of Energy, under Contract No. DE-AC03-76SF00098. 


\section{ABSTRACT}

In this paper we extend our previous analysis of cold beam tube vacuum in a superconducting proton collider to include ion desorption in addition to thermal desorption and synchrotron radiation induced photodesorption. The new ion desorption terms introduce the possibility of vacuum instability. This is similar to the classical room temperature case but now modified by the inclusion of ion desorption coefficients for cryosorbed (physisorbed) molecules which can greatly exceed the coefficients for tightly bound molecules. The sojourn time concept for physisorbed $\mathrm{H}_{2}$ is generalized to include photodesorption and ion desorption as well as the usually considered thermal desorption. The ion desorption rate is density dependent and divergent so at the onset of instability the sojourn time goes to zero. Experimental data are used to evaluate the $\mathrm{H}_{2}$ sojourn time for the conditions of the Large Hadron Collider (LHC) and the situation is found to be stable. The sojourn time is dominated by photodesorption for surface density $s\left(H_{2}\right)$ less than a monolayer and by thermal deposition for $s\left(\mathrm{H}_{2}\right)$ greater than a monolayer. For a few per cent of a monolayer, characteristic of a beam screen, the photodesorption rate exceeds ion desorption rate by more than two orders of magnitude. The photodesorption rate corresponds to a sojourn time of approximately $100 \mathrm{sec}$. The paper next turns to the evaluation of stability margins and inclusion of gases heavier than $\mathrm{H}_{2}\left(\mathrm{CO}, \mathrm{CO}_{2}\right.$ and $\left.\mathrm{CH}_{4}\right)$, where ion desorption introduces coupling between molecular species. Stability conditions are worked out for a simple cold beam tube, a cold beam tube pumped from the ends and a cold beam tube with a co-axial perforated beam screen. In each case a simple inequality for stability of a single component is replaced by a determinant that must be greater than zero for a gas

mixture. The connection with the general theory of feedback stability is made and it is shown that the gains of the diagonal uncoupled feedback loops are first order in 
the ion desorption coefficients whereas the gains of the off diagonal coupled feedback loops are second and higher order. For this reason it turns out that in practical cases stability is dominated by the uncoupled diagonal elements and the inverse of the largest first order closed loop gain is a useful estimate of the margin of stability. In contrast to the case of a simple cold beam tube, the stability condition for a beam screen does not contain the desorption coefficient for physisorbed molecules, even when the screen temperature is low enough that there is a finite surface density of them on the screen surface. Consequently there does not appear to be any particular advantage to operating the beam screen at high enough temperature to avoid physisorption. Numerical estimates of ion desorption stability are given for a number of cases relevant to LHC and all of the ones likely to be encountered were found to be stable. The most important case, a $1 \%$ transparency beam screen at $\sim 4.2 \mathrm{~K}$, was found to have a stability safety margin of approximately thirty determined by ion desorption of $\mathrm{CO}$. Ion desorption of $\mathrm{H}_{2}$ is about a factor of eighty less stringent than $\mathrm{CO}$. For these estimates the beam tube surface was assumed to be solvent cleaned but otherwise untreated, for example by a very high temperature vacuum bakeout or by glow discharge cleaning. 


\section{INTRODUCTION AND BASIC EQUATIONS}

Following our previous analysis, 1,2 three equations are introduced to describe the evolution of $\mathrm{H}_{2}$ molecules in the beam tube of a superconducting proton collider, assumed to have energy high enough that synchrotron radiation desorption must be taken into account; e.g. the CERN Large Hadron Collider (LHC) with $\dot{\Gamma}=10^{17}$ photons $/ \mathrm{m} / \mathrm{sec}, E_{c r i t}=46 \mathrm{eV}$ and radiated power per beam $d P / d z=0.22 \mathrm{~W} / \mathrm{m} .{ }^{3}$ The first equation describes the gas density $(n)$ in the beam tube, the second the surface density $(s)$ of physisorbed molecules and the third the isotherm density $\left(n_{e}\right)$ due to the physisorbed molecules. These are given by eqns. (1) to (3) below;

$$
\begin{gathered}
V \frac{\partial n}{\partial t}=\eta_{i} \frac{I \sigma_{p i}}{e} n+\eta_{i}^{\prime} \frac{I \sigma_{p i}}{e} n+\eta_{1} \dot{\Gamma}+\eta^{\prime} \dot{\Gamma}-\sigma_{w} S_{w} n+\frac{A_{w} s}{\tau_{w}^{t}}-C^{*} n+A_{c} D \frac{\partial^{2} n}{\partial z^{2}} \\
A_{w} \frac{\partial s}{\partial t}=\eta_{2} \dot{\Gamma}+\sigma_{w} S_{w} n-\frac{A_{w} s}{\tau_{w}^{t}}-\eta^{\prime} \dot{\Gamma}-\eta_{i}^{\prime} \frac{I \sigma_{p i}}{e} n \\
\log \left(\frac{s}{s_{m}}\right)=-B\left[k T^{*} \log \left(\frac{n_{e}}{n_{s a t}}\right)\right]^{2}
\end{gathered}
$$

where $V=\pi r_{w}{ }^{2}$ is the beam tube volume and $A_{w}=2 \pi r_{w}$ the beam tube surface area, both per unit axial length. The first two terms on the right side of eqn. (1) are the new ion desorption terms added to the earlier equations. ${ }^{1}$ The first term refers to tightly bound $\mathrm{H}_{2}$ not previously desorbed; $I$ is the proton beam current, $\sigma_{p i}$ is the cross section for ionization of $\mathrm{H}_{2}$ by a beam proton and $\eta_{i}(\geq-1)$ is the net number of molecules desorbed per incident ion accelerated into the wall by the beam space charge force. If $\eta_{i}$ is negative then the ionization of molecules actually serves as a pump. Ions adsorbed in the wall are assumed not to contribute to the surface density of physisorbed molecules; if they all did there would be an additional term $\left(I \sigma_{p i} / e\right) n$ on the right side of eqn. 2. The second term is for desorption of relatively weakly bound physisorbed molecules with coefficient $\eta_{i}^{\prime}$. For low surface coverage $\eta_{i}^{\prime}$ depends linearly on $s ; \eta_{i}^{\prime}=\eta_{i 0}^{\prime}\left(s / s_{m}\right)$ where $s_{m}$ 
is the monolayer density. Whereas for an approximately $500 \mathrm{eV} \mathrm{H}_{2}+$ ion, $\eta_{i}$ is of order unity for a clean but otherwise unbaked and unconditioned metal surface, ${ }^{4} \eta_{i}^{\prime}$ can be very large. ${ }^{5}$ The data in ref. 5 for $500 \mathrm{eV} \mathrm{H}_{2}{ }^{+}$can be fit with $\eta_{i 0}^{\prime}=1500$ and the linear approximation is valid up to $s / s_{m} \approx 3$, or $s \sim 10^{16} \mathrm{H}_{2} / \mathrm{cm}^{2}$. Between $s=10^{16}$ and $10^{18} \mathrm{H}_{2} / \mathrm{cm}^{2}$ the ion desorption coefficient reaches a broad plateau and then decreases roughly two orders of magnitude until $s \sim$ $10^{19} \mathrm{H}_{2} / \mathrm{cm}^{2}$ and then increases relatively slowly. The shape of the desorption curve appears nearly independent of the ion energy and shifts upward with increasing energy. It is the large magnitude of $\eta_{i}^{\prime}$ which prompted the investigation described in this paper and the clarification of its role in the ion desorption stability of superconducting proton colliders. The third term in eqn. 1 describes photodesorption of tightly bound molecules with coefficient $\eta_{1}$ and photon intensity per unit length $\dot{\Gamma}$, the fourth term is for photodesorption of physisorbed $\mathrm{H}_{2}$ with coefficient $\eta^{\prime}=\eta_{O}^{\prime}\left(s / s_{m}\right)$, the fifth term represents pumping by the beam tube surface with sticking coefficient $\sigma_{w}$ and ideal wall pumping speed $S_{w}=\bar{v} A_{w} / 4$, the sixth term is due to the isotherm evaporation of molecules with thermal sojourn time $\tau_{w}^{t}$, the seventh term represents distributed pumping where $C$ is the pumping speed per unit length of the perforations in a coaxial liner or beam screen, if present, and the last term is due to axial diffusion and discrete pumping with $D=$ (2/3) $r_{w}$ and $A_{c}=\pi r_{w}{ }^{2}$. The linear dependence of $\eta^{\prime}$ on $s$ is known to be valid up to at least one monolayer $s_{m}=3 \times 10^{15} \mathrm{H}_{2} / \mathrm{cm}^{2}{ }^{2}$ The first term in eqn. 2 allows for the direct production of physisorbed molecules by photodesorption of tightly bound molecules with coefficient $\eta_{2}$. The remaining four terms account for adsorption and desorption of physisorbed molecules and have their counterparts with opposite sign in eqn. 1. For the $\mathrm{H}_{2}$ isotherm we have taken a DRK equation 7 which gives a better fit to experimental data than the BET equation we have used earlier, particularly in the region $s / s_{m}<1$. The hydrogen adsorption data on stainless steel at $4.2 \mathrm{~K}$ in ref. 8 have been fit with $B-0.5=5.2 \mathrm{meV} /$ molecule and $s_{m}=3.0 \times 10^{15} \mathrm{H}_{2} / \mathrm{cm}^{2}$. The saturation density of $\mathrm{H}_{2}$ at $4.2 \mathrm{~K}$ is $n_{\text {sat }}=2.0 \times 10^{12} \mathrm{H}_{2} / \mathrm{cm}^{3}$.

Equations similar to (1) and (2) can be written for other gases; $\mathrm{CO}, \mathrm{CO}_{2}$ and $\mathrm{CH}_{4}$. For all gases but $\mathrm{H}_{2}$ and $\mathrm{He}$, the isotherm density is negligible for the temperatures of interest $(<20 \mathrm{~K})$. 
For multi-component gas mixtures the ion desorption terms introduce coupling between molecular species $m_{k}=\left\{\mathrm{H}_{2}, \mathrm{CO}, \mathrm{CO}_{2}, \mathrm{CH}_{4} ; k=1,4\right\}$ so for example the first term of eqn. 1 for species $n_{j}$ is replaced by;

$$
\sum_{k} \eta_{i}\left(m_{k}^{+}, m_{j}\right)\left(\frac{I \sigma_{p i}\left(m_{k}\right)}{e}\right) n_{k}
$$

where $\eta_{i}\left(m_{k}^{+}, m_{j}\right)$ is the ion desorption coefficient of molecular species $m_{j}$ by ion species $m_{k}^{+}$ and $\sigma_{p i}\left(m_{k}\right)$ is the proton ionization cross section for $m_{k}$.

From the form of eqn. (1) we can define a generalized sojourn time $\tau_{w}$ due to thermal, photon and ion desorption of physisorbed molecules which is valid in the region of linear dependence of $\eta^{\prime}$ and $\eta_{i}^{\prime}$ on s. It is given by;

$$
\begin{aligned}
\frac{1}{\tau_{w}} & =\frac{1}{\tau_{w}^{t}}+\frac{1}{\tau_{w}^{\eta^{\prime}}}+\frac{1}{\tau_{w}^{\eta_{i}^{\prime}}} \\
& =\frac{\sigma_{w}^{t} \bar{v}_{t}}{4} \frac{n_{e}}{s}+\frac{\eta_{0}^{\prime} \dot{\Gamma}}{A_{w} s_{m}}+\frac{\eta_{i 0}^{\prime} *\left(\frac{I \sigma_{p i}}{e} n\right)}{A_{w} s_{m}}
\end{aligned}
$$

For ion desorption instability initiated by physisorbed molecules the density $n$ goes to infinity and the sojourn time to zero.

\section{SOLUTIONS AND STABILITY BOUNDS}

In this section we present the solutions and stability bounds to eqns. 1 to 3 for three interesting cases: $(A)$ a beam tube long enough that axial diffusion may be neglected, $(B)$ a beam tube short enough that axial diffusion must be included and $(C)$ a beam tube with a coaxial perforated beam screen. In all cases we are interested in time scales that are long compared to a molecular transit 
time across the tube diameter and therefore $V \frac{\partial n}{\partial t} \approx 0$. In the second and third cases we also look for the solutions with the surface in quasi steady state $A_{w} \frac{\partial s}{\partial t} \approx 0$.

A. Long beam tube without a beam screen; $v \frac{\partial n}{\partial t} \approx 0, C=0, A_{c} D \frac{\partial^{2} n}{\partial z^{2}} \approx 0$

In this case the solution is;

$$
\begin{aligned}
& n=\frac{\eta_{1} \dot{\Gamma}+\eta^{\prime} \dot{\Gamma}+\frac{A_{w} s}{\tau_{w}^{t}}}{\sigma_{w} S_{w}-\eta_{i} \frac{I \sigma_{p i}}{e}-\eta_{i}^{\prime} \frac{I \sigma_{p i}}{e}} \\
& s=\frac{1}{A_{w}} \int_{0}^{\Gamma}\left[\eta_{1}+\eta_{2}+\left(\frac{I \sigma_{p i}}{e \dot{\Gamma}} n\right) \eta_{i}\right] d \Gamma
\end{aligned}
$$

and in accordance with our assumed conditions $\lambda^{2}=\frac{A_{c} D}{\sigma_{w} S_{w}}=\frac{4}{3} \frac{r_{w}{ }^{2}}{\sigma_{w}} \ll L^{2}$. From the numerator of eqn. 6 we identify the three components of $H_{2}$ density due to (1) photodesorption of tightly bound molecules, (2) photodesorption of physisorbed molecules and (3) thermal desorption. Ion desorption behaves like a multiplier and increases the density of each component by a factor $\sigma_{w} S_{w} /\left(\sigma_{w} S_{w}-\eta_{i}\left(\frac{I \sigma_{p i}}{e}\right)-\eta_{i}^{\prime}\left(\frac{I \sigma_{p i}}{e}\right)\right)$. From the denominator of eqn. 6 the density remains stable and finite only if the following inequality is obeyed;

$$
\left(\eta_{i}+\eta_{i}^{\prime}\right) I<\frac{e \sigma_{w} S_{w}}{\sigma_{p i}}
$$

Eqn. 8 is similar to the classical room temperature ion desorption stability requirement discovered in the context of the CERN ISR many years ago ${ }^{9}$ but now augmented by the adsorption and desorption of physisorbed molecules. The stability requirement is equivalent to the statement that 
the net gain in production of neutral molecules by ionization, desorption and wall pumping must be less than unity. A feedback diagram illustrating the solution given by eqn. 6 is shown in Fig. 1 . The loop gain $G$ is

$$
G=\frac{\left(\eta_{i}+\eta_{i}^{\prime}\right) \frac{I \sigma_{p i}}{e}}{\sigma_{w} S_{w}}
$$

and $G<1$ is required for a stable $H_{2}$ density. Since ion desorption appears only in the denominator of eqn. 6 it behaves like an amplifier multiplying the other sources of gas that are present. For this reason ion desorption needs some other source of gas in order to come into play. The surface density of physisorbed $\mathrm{H}_{2}$ builds up steadily according to eqn. 7 due to adsorption of the tightly bound hydrogen that has been desorbed by photon and ion impact. This eventually will lead to large increases in the density due to the $\eta^{\prime}$ and isotherm terms in the numerator of eqn. 6 and may destabilize the denominator. The stability requirement in eqn. 8 is independent of the sources of gas in the numerator of eqn. 6 . Although we mainly have in mind situations where photodesorption is significant, eqn. 8 applies to any cold beam tube situation, in particular to regions where there may be a cold beam tube but no significant photon flux and therefore no beam screen - e.g. superconducting quadrupoles in a long insertion region straight section of LHC, the existing $1 \mathrm{TeV}$ superconducting proton storage rings at FNAL and DESY etc.. In these cases one does not expect the surface density of physisorbed molecules to build up at a significant rate and the beam tube vacuum density should be very low.

The stability bound in eqn. 8 has been derived for a single component. As noted in eqn. 4 , ion desorption has the interesting possibility of coupling molecular species. It is straightforward to work out the stability requirement for a multi-component gas mixture, e.g. $\left\{m_{j} ; j=1,4\right\}$ for $\left\{H_{2}\right.$, $\left.\mathrm{CO}, \mathrm{CO}_{2}, \mathrm{CH}_{4}\right\}$. Eqn. 8 is replaced by $\operatorname{det}\left(A_{j k}\right)=\operatorname{det}\left(\delta_{j k}-R_{j k}\right)>0$ where 


$$
\begin{aligned}
A_{j j} & =1-\frac{\left(\eta_{i}\left(m_{j}^{+}, m_{j}\right)+\eta_{i}^{\prime}\left(m_{j}^{+}, m_{j}\right)\right)}{\sigma_{w}\left(m_{j}\right) S_{w}\left(m_{j}\right)} \times \frac{I \sigma_{p i}\left(m_{j}\right)}{e} \\
& =1-R\left(m_{j}^{+}, m_{j}\right)
\end{aligned}
$$

and for $j \neq k$,

$$
\begin{aligned}
A_{j k} & =-\frac{\left(\eta_{i}\left(m_{k}^{+}, m_{j}\right)+\eta_{i}^{\prime}\left(m_{k}^{+}, m_{j}\right)\right)}{\sigma_{w}\left(m_{j}\right) S_{w}\left(m_{j}\right)} \times \frac{I \sigma_{p i}\left(m_{k}\right)}{e} . \\
& =-R\left(m_{k}^{+}, m_{j}\right)
\end{aligned}
$$

A feedback diagram illustrating the solution for a two component $\mathrm{H}_{2}, \mathrm{CO}$ system is given in Fig. 2. In addition to the first order feedback loops for each species, there is now a second order loop coupling the two species. In the stability condition $\operatorname{det}\left(A_{j k}\right)>0$, the first order loops are contained in the product of the diagonal elements and the second order loop in the product of the off diagonal elements. The three loop gains may be read directly from Fig. 2;

$$
\begin{aligned}
& G\left(\mathrm{H}_{2}\right)=\frac{\left(\eta_{i}\left(\mathrm{H}_{2}^{+}, \mathrm{H}_{2}\right)+\eta_{i}^{\prime}\left(\mathrm{H}_{2}^{+}, \mathrm{H}_{2}\right)\right)}{\sigma_{w}\left(\mathrm{H}_{2}\right) S_{w}\left(\mathrm{H}_{2}\right)} \times \frac{I \sigma_{p i}\left(\mathrm{H}_{2}\right)}{e}, \\
& G(\mathrm{CO})=\frac{\left(\eta_{i}\left(\mathrm{CO}^{+}, \mathrm{CO}\right)+\eta_{i}^{\prime}\left(\mathrm{CO}^{+}, \mathrm{CO}\right)\right)}{\sigma_{w}(\mathrm{CO}) S_{w}(\mathrm{CO})} \times \frac{I \sigma_{p i}(\mathrm{CO})}{e},
\end{aligned}
$$

and

$$
\begin{aligned}
G\left(\mathrm{H}_{2}, \mathrm{CO}\right)=\frac{\left(\eta_{i}\left(\mathrm{H}_{2}^{+}, \mathrm{CO}\right)+\eta_{i}^{\prime}\left(\mathrm{H}_{2}^{+}, \mathrm{CO}\right)\right)}{\sigma_{w}(\mathrm{CO}) S_{w}(\mathrm{CO})} \times \frac{I \sigma_{p i}\left(\mathrm{H}_{2}\right)}{e} \\
\times \frac{\left(\eta_{i}\left(\mathrm{CO}^{+}, \mathrm{H}_{2}\right)+\eta_{i}^{\prime}\left(\mathrm{CO}^{+}, \mathrm{H}_{2}\right)\right)}{\sigma_{w}\left(\mathrm{H}_{2}\right) S_{w}\left(\mathrm{H}_{2}\right)} \times \frac{I \sigma_{p i}(\mathrm{CO})}{e} .
\end{aligned}
$$


The loop gains are simply related to the matrix elements of $\mathrm{Rjk}_{\mathrm{j}} \mathrm{G}\left(\mathrm{H}_{2}\right)=R\left(\mathrm{H}_{2}{ }^{+}, \mathrm{H}_{2}\right), G(\mathrm{CO})=$ $\left.\mathrm{R}\left(\mathrm{CO}^{+}, \mathrm{CO}\right), \mathrm{G}\left(\mathrm{H}_{2}, \mathrm{CO}\right)=\mathrm{R}_{\left(\mathrm{H}_{2}\right.}{ }^{+}, \mathrm{CO}\right) \times R\left(\mathrm{CO}^{+}, \mathrm{H}_{2}\right)$. This procedure is readily extended to an arbitrary number of species. For three species there are three first order loops, three second order loops and two third order loops etc.. A necessary condition for stability is that each closed loop gain be less than one, a sufficient condition is that the sum of the closed loop gains be less than one. Due to coupling it is possible to have instability even if none of the closed loops taken individually is unstable. For example in the two dimensional case $R_{11}=R_{22}=R_{12}=R_{21}=0.5$ has $\operatorname{det}(I-R)=0$. When the off diagonal elements can be neglected in $\operatorname{det}(I-R)$ then the system is effectively decoupled and instability can be reached only when at least one of the diagonal elements is less than or equal to zero. In this case a useful single number characterizing the stability margin can be defined and is simply the inverse of the largest diagonal element of $R$. It is the factor by which the product of ion desorption coefficient and beam current would have to increase to produce an instability. Intuitively perhaps one expects instability to be dominated by one of the first order loops however even in this situation it is possible to imagine subtle situations. For example a build up of physisorbed $\mathrm{CO}$ due to $\mathrm{H}_{2}{ }^{+}$ion bombardment which eventually becomes high enough to destabilize the first order $\mathrm{CO}$ loop even though the $\mathrm{H}_{2}$ and second order $\left(\mathrm{H}_{2}, \mathrm{CO}\right)$ loops remain stable.

\section{B. Short beam tube without a beam screen; $v \frac{\partial n}{\partial t} \approx 0, C=0, A \frac{\partial s}{\partial t} \approx 0$}

A beam tube short enough that axial diffusion to the ends is significant will reach a steady state characterized by a constant surface density of physisorbed molecules and a balance between the desorption rate of tightly bound molecules and the quantity of gas pumped out the ends of the tube. We assume that discrete pumping at $\pm L / 2$ is strong enough that the density at the ends is less than anywhere else in the tube. In this case the beam tube density is given by; 


$$
n=\frac{\left(\eta_{1}+\eta_{2}\right) \dot{\Gamma}}{\eta_{i} \frac{I \sigma_{p i}}{e}}\left(\frac{\cos \left(\frac{z}{\lambda_{\eta_{i}}}\right)}{\cos \left(\frac{L}{2 \lambda_{\eta_{i}}}\right)}-1\right)+\frac{\cos \left(\frac{z}{\lambda_{\eta_{i}}}\right)}{\cos \left(\frac{L}{2 \lambda_{\eta_{i}}}\right)} n\left( \pm \frac{L}{2}\right)
$$

and the surface density by;

$$
s=\frac{\eta_{2} \dot{\Gamma}+\sigma_{w} s_{w} n}{\eta_{0}{ }^{\prime} \dot{\Gamma}+\eta_{i 0}{ }^{\prime}\left(\frac{I \sigma_{p i}}{e} n\right)+\frac{A_{w} s_{m}}{\tau_{w}^{t}}} s_{m}
$$

where $\frac{1}{\lambda_{\eta_{i}}^{2}}=\frac{\eta_{i}}{A_{c} D} \times \frac{I \sigma_{p i}}{e}$. One can easily check that as ion desorption goes to zero, or $\lambda_{\eta_{i}} \rightarrow \infty$, eqn. 12 goes over to the usual parabolic solution for a tube with a uniform gas source. From eqn. 12 , the density in a beam tube short enough to have reached surface equilibrium due to pumping at the ends remains finite only if the following inequality holds;

$$
\frac{L}{2 \lambda_{\eta_{i}}}<\frac{\pi}{2}
$$

or,

$$
\begin{aligned}
& \eta_{i} I<\pi^{2} \frac{e}{\sigma_{p i}} \frac{A_{c} D}{L^{2}} \\
& \eta_{i} I<\pi^{2} \frac{e}{\sigma_{p i}}\left(\frac{C_{L}}{L}\right)
\end{aligned}
$$

where $C_{L}=\frac{A_{C} D}{L}$ is the conductance of a tube of length $L$ and radius $r_{w}$. This is the same as the stability condition ordinarily encountered in room temperature, conductance limited beam tubes, the reason for this being that the surface is in steady state and effectively not pumping. In the low temperature case considered here, even though there is a finite surface density of physisorbed molecules given by eqn. $10, \eta_{i}{ }^{\prime}$ and $\eta^{\prime}$ do not appear in eqn. 12 for density and $\eta_{i}{ }^{\prime}$ does not appear in the stability condition. Furthermore according to eqn. 13 the equilibrium surface density 
is less in the presence of ion desorption than without it. We will meet this situation again in the cold beam tube with a screen. A feedback diagram similar to Fig. 1 applies with $\eta_{i}^{\prime}=0$ and $1 / \sigma_{w} S_{w}$ replaced by $\pi^{2} C_{L} / L$. The loop gain $G$ is;

$$
G=\frac{\eta_{i} \frac{I \sigma_{p i}}{e}}{\pi^{2} \frac{C_{L}}{L}}
$$

where according to eqn. $14, G<1$ is required for stability.

The multi-component generalization of eqn. 14 may be worked out by introducing separate eqns. 1 and 2 for each molecular species and the ion desorption coupling term given by eqn. 4 . The equations are then Fourier transformed to convert the diffusion terms from differential to algebraic expressions, obtaining the following equation for each species $m_{r}$;

$$
-k^{2} \tilde{n}_{r}+\frac{1}{A_{c} D\left(m_{r}\right)} \sum_{s} \eta_{i}\left(m_{s}^{+}, m_{r}\right) \frac{I \sigma_{p i}\left(m_{s}\right)}{e} \tilde{n}_{s}=-\frac{\eta_{1}\left(m_{r}\right)+\eta_{2}\left(m_{r}\right)}{A_{c} D\left(m_{r}\right)} \dot{\Gamma}
$$

where $\tilde{n}_{r}$ is the Fourier transformed density. For stability the determinant of coefficients on the left side of eqn. 16 must not vanish. Small $k$ or long wavelength reaches instability first. The longest permissible wavelength for a tube of length $L$ is $2 L$. Inserting $k=\pi / L$ in eqn. 16 then the condition for stability is $\operatorname{det}\left(A_{j k}\right)>0$ where;

$$
\begin{aligned}
A_{j j} & =1-\frac{\eta_{i}\left(m_{j}^{+}, m_{j}\right)}{\pi^{2} \frac{A_{c} D}{L^{2}}} \times \frac{I \sigma_{p i}\left(m_{j}\right)}{e} \\
& =1-R\left(m_{j}^{+}, m_{j}\right)
\end{aligned}
$$

and for $\mathrm{j} \neq \mathrm{k}$, 


$$
\begin{aligned}
A_{j k} & =-\frac{\eta_{i}\left(m_{k}^{+}, m_{j}\right)}{\pi^{2} \frac{A_{c} D}{L^{2}}} \times \frac{I \sigma_{p i}\left(m_{k}\right)}{e} . \\
& =-R\left(m_{k}^{+}, m_{j}\right)
\end{aligned}
$$

Expressions analogous to eqns. 11 may again be written for the loop gains; for first order $G\left(m_{j}\right)=$ $R\left(m_{j}{ }^{+}, m_{j}\right)$, for second order $j \neq k G\left(m_{j}, m_{k}\right)=R\left(m_{k}{ }^{+}, m_{j}\right) \times R\left(m_{j}{ }^{+}, m_{k}\right)$.

C. Beam tube with a beam screen; $C n>>A_{c} D \partial^{2} n / \partial z^{2}, v \frac{\partial n}{\partial t} \approx 0, A \frac{\partial s}{\partial t} \approx 0$

In this case the density is given by;

$$
n=\frac{\left(\eta_{1}+\eta_{2}\right) \dot{\Gamma}}{C-\eta_{i} \frac{I \sigma_{p i}}{e}}
$$

and the equation for surface density is exactly the same as eqn. 13. From the denominator of eqn. 18 we have the ion desorption stability condition;

$$
\eta_{i} I<\frac{e C}{\sigma_{p i}}
$$

Comparing eqn. 19 to the stability of a simple beam tube eqn. 8, the desorption coefficient $\eta_{i}^{\prime}$ for physisorbed molecules no longer appears on the left side whereas on the right side the pumping speed of the entire wall $\sigma_{w} S_{w}$ has been replaced by the conductance of the beam screen perforations which at most cover a few per cent of the wall surface. Again ion desorption behaves like a multiplier, increasing the density by a factor $C /\left(C-\eta_{i}\left(\frac{I \sigma_{p i}}{e}\right)\right)$ compared to what it would be in the absence of ion desorption.

As with the earlier cases, the stability condition for a multi-component gas mixture is replaced by a condition on a determinant, $\operatorname{det}\left(A_{j k}\right)>0$ where; 


$$
\begin{aligned}
A_{j j} & =1-\frac{\eta_{i}\left(m_{j}^{+}, m_{j}\right)}{C\left(m_{j}\right)} \frac{I \sigma_{p i}\left(m_{j}\right)}{e} \\
& =1-R\left(m_{j}^{+}, m_{j}\right)
\end{aligned}
$$

and for $j \neq k$,

$$
\begin{aligned}
& A_{j k}=-\frac{\eta_{i}\left(m_{k}^{+}, m_{j}\right)}{C\left(m_{j}\right)} \frac{I \sigma_{p i}\left(m_{k}\right)}{e} . \\
& =-R\left(m_{k}^{+}, m_{j}\right)
\end{aligned}
$$

The relationships between the loop gains and $R\left(m_{k}{ }^{+}, m_{j}\right)$ matrix elements are the same as for the previously given cases.

\section{NUMERICAL EXAMPLES OF BEAM TUBE $\mathrm{H}_{2}$ DENSITY AND SOJOURN TIME}

In Fig. 3 we have plotted the solutions for $\mathrm{H}_{2}$ density for the three cases given by eqns. 6, 12 and 18 versus the integrated photon flux $\Gamma$. Ion desorption is neglected and this will be justified aposteriori below. These type of solutions have been described in detail previously. ${ }^{2}$ We deal with them briefly here to set the context for discussing ion desorption. For these calculations we have assumed conditions close to the beam tube arcs of the LHC, $\dot{\Gamma}=10^{17}$ photons $/ \mathrm{m} / \mathrm{sec}$, beam tube effective radius $r_{w}=2.3 \mathrm{~cm}$, photodesorption coefficients $\eta=\eta_{1}+\eta_{2}=10^{-3}$ for tightly bound $H_{2}$ and $\eta^{\prime} \mathrm{O}=0.525$ for physisorbed $\mathrm{H}_{2},{ }^{6}$ a beam tube temperature $4.2 \mathrm{~K}$ corresponding to the beam screen rather than the $1.8 \mathrm{~K}$ magnet bore tube. The isotherm parameters have been given in Sec. I. The question of what to take for the sticking coefficients and the molecular velocities is always somewhat nettlesome. Here we take $\bar{v}=8 \times 10^{4} \mathrm{~cm} / \mathrm{sec}, \sigma_{w}=.075$ for photodesorbed molecules 10 and $\bar{v}_{t}=2.1 \times 10^{4} \mathrm{~cm} / \mathrm{sec}, \sigma_{w}^{t}=1.0$ for thermally desorbed molecules. Since it is the product $\sigma \times \bar{v}$ which appears in the wall pumping term the calculations are not too sensitive to this 
distinction. For simplicity the equations in Secs. I and II have been written with only a single velocity characterizing beam tube molecules. The prescription for dealing with multiple velocity components in the calculations of Figs. 3 and 4 is described in Appendix I.

Before describing the solutions in Fig. 3 we remark that the desired vacuum limited luminosity lifetime in LHC of $>100 \mathrm{hrs}$. sets an upper bound on the average beam tube density of $<10^{9} \mathrm{H}_{2} / \mathrm{cm}^{3}$ due to beam gas scattering. The local density limit $<3 \times 10^{10} \mathrm{H}_{2} / \mathrm{cm}^{3}$ is set by a requirement of less than $1 \mathrm{~W} / \mathrm{m}$ scattered beam power absorbed in the magnet cryostats.

The solid line in Fig. 3 is the solution for $\mathrm{H}_{2}$ density for an infinitely long beam tube given by eqn. 6 and is the sum of the three labeled components due to (1) desorption of tightly bound $\mathrm{H}_{2}$, (2) desorption of physisorbed $\mathrm{H}_{2}$ and (3) thermal desorption. Four solutions for $\mathrm{H}_{2}$ density at the center of a finite length beam tube given by eqn. 12 are labeled by the tube length $L=1,5,15$ and $50 \mathrm{~m}$. The last three lengths correspond to the approximate lengths of LHC components: a short straight section (containing a quadrupole and correction magnets), a dipole bending magnet and a half cell (three dipoles plus a short straight section). For these calculations we have assumed ideal pumping at the ends that is large compared to the beam tube conductance. A single solution to eqn. 18 for a beam screen is labeled with the assumed effective transparency of perforations " $1 \%$ " for pumping desorbed molecules. All beam tubes initially obey the infinite length approximation and follow the solid curve until they intersect the appropriate finite length solution or beam screen solution if it is present. The surface density of physisorbed $H_{2}$ has reached $s / s_{m}=.014$ when the infinite length solution intersects the "1\%" beam screen solution. In the case of the $50 \mathrm{~m}$ beam tube at $4.2 \mathrm{~K}$ the finite length solution exceeds the $\mathrm{H}_{2}$ saturation density so the solution remains on the infinite length case for the duration of the calculation shown in Fig. 3. The necessity of distributed pumping is clearly evident in Fig. 3.

The question sometimes arises whether the beam screen can be left out of certain isolated sections, for example dispersion suppressors or interaction region quadrupoles, where the betatron function may be large and it is desirable not to increase the magnet coil aperture more than necessary. The deciding factor is the maximum locally scattered beam power that can be tolerated 
and the answer depends on the details of photon intensity, the beam tube length and radius, and the magnitude of photodesorption coefficients for tightly bound molecules after reasonable beam conditioning. For the assumed conditions in the arcs of LHC and Fig. 3, the maximum length tube that could be pumped from the ends is $L \approx 5 \mathrm{~m}$ due to scattering by $\mathrm{H}_{2}$ alone. Including scattering by the other photodesorbed gases, primarily $\mathrm{CO}$ and $\mathrm{CO}_{2}$, increases the $\mathrm{H}_{2}$ equivalent desorption coefficient of tightly bound molecules (and therefore the scattered beam power) by approximately a factor of ten. Other situations can be evaluated similarly when the details are specified.

In Fig. 4 we have plotted the sojourn time of a physisorbed $\mathrm{H}_{2}$ molecule given by eqn. 5 as a function of surface density $s$ up to two monolayers. The three components due to (1) thermal desorption, (2) photon desorption and (3) ion desorption are shown by dashed lines. The parameters assumed for this calculation are the same as given previously for Fig. 3. For ion desorption we have taken $\eta^{\prime} i \mathrm{O}=1500$ from ref. 5 for $500 \mathrm{eV} \mathrm{H}_{2}{ }^{+}$incident on a layer of physisorbed $H_{2}$. For surface density less than a monolayer $s_{m}=3 \times 10^{15} \mathrm{H}_{2} / \mathrm{cm}^{2}$ the sojourn time is approximately $100 \mathrm{sec}$, independent of $s$ and dominated by photodesorption. As one monolayer is approached the sojourn time due to thermal desorption decreases rapidly and becomes the dominant factor, reaching approximately $0.5 \mathrm{sec}$ between one and two monolayers. The sojourn time due to ion desorption is always greater than the resultant by more than one order of magnitude. In the region of a few per cent of a monolayer, which is of most interest for a beam screen, this ratio increases to more than two orders of magnitude. For this reason the effects of ion desorption are not visible on a plot like Fig. 3. The actual ion desorption stability safety margins are discussed in the next section.

Before turning to the numerical estimates of stability in the next section it is useful to discuss in a qualitative way what can happen when instability occurs. The infinite length cold beam tube and $1 \%$ transparency beam screen solutions from Fig. 3 have been reproduced in Fig. 5 together with hypothetical examples of the influence of ion desorption. First we examine the beam screen case and observe from eqn. 18 that as ion desorption becomes more important it simply shifts the solution, and therefore the intersection with the infinite length case, to higher density. This is seen 
in Fig. 5 for an example with $\eta_{i} \frac{I \sigma_{p i}}{e} / C=0.9$, which has increased the beam screen density one order of magnitude. As the beam screen gets closer to instability the solution moves up in density until it eventually vanishes from Fig. 5 and the infinite length solution pertains either indefinitely or until it becomes unstable. For the infinite length case increasing the magnitude of ion desorption causes a singularity to move in from the right side of Fig. 5. For the example in Fig. 5 the singularity occurs at $\Gamma=4 \times 10^{20}$ photons $/ \mathrm{m}$, or $\left(\eta_{i}+\eta_{i}^{\prime}\right) \frac{I \sigma_{p i}}{e} / \sigma_{w} s_{w}=1$ at $s / s_{m} \sim 0.1$. As long as a stable beam screen solution exists, the effect of ion desorption and instability on the infinite length solution is to move the intersection with the beam screen solution to lower integrated photon flux - i.e. the beam screen solution simply pertains sooner than it would have in the absence of ion desorption. The intersections are indicated by the black dots on Fig. 5. This behavior of the beam screen is reminiscent of what happened previously with inclusion of the photodesorption of physisorbed gas. Although introducing $\eta^{\prime} \dot{\Gamma}$ in eqn. 1 cannot lead to an instability, it moves the intersection of the infinite length and beam screen solutions to lower integrated photon flux. In general factors which cause the infinite length density to increase while leaving the beam screen density alone simply hasten the time when the beam screen solution takes over. We emphasize that the examples in Fig. 5 have been chosen to magnify the effect of ion desorption for illustration and appear to correspond to situations far from reality. In addition to the usual LHC parameters the cases (3) and (4) in Fig. 5 correspond to $\eta_{i}=1,000$ and $\eta^{\prime} i 0=1.2 \times 10^{6}$ respectively, about three orders of magnitude higher than what seem to be experimentally reasonable values of these ion desorption coefficients.

\section{NUMERICAL ESTIMATES OF ION DESORPTION STABILITY MATRICES}

In this section we give numerical estimates of ion desorption stability margins for cases that have practical importance for the LHC. Mainly the estimates will be restricted to the two 
component $\left(\mathrm{H}_{2}, \mathrm{CO}\right)$ system due to limitations of experimental data. This of course isn't accidental. Hydrogen generally has the highest photon and ion desorption coefficients of all the gases. Carbon monoxide is next and allows estimating the effects of coupling and of a molecular mass heavier than hydrogen. The discussion follows the order of the previous section. We first evaluate the stability matrix given by eqn. 10 for the infinitely long cold beam tube approximation with the surface of the tube pumping. As we have seen in Fig. 3, when synchrotron radiation is present at the expected intensity in LHC, photodesorption requires a beam screen or discrete pumping at rather short intervals. Consequently this case is mostly interesting for the initial exposure to photons, before the surface has reached a steady state due to beam screen or discrete pumping. Furthermore, as we have seen in Fig. 5, if a beam screen is present and instability of this type occurs, it simply hastens the transition to the beam screen solution with the surface of the beam screen in quasi equilibrium and no longer pumping. The absolute upper bound $\mathrm{H}_{2}$ surface density $s$ of interest, which appears in eqn. 10 through $\eta_{i}^{\prime}(s)=\eta_{i 0}^{\prime}\left(s / s_{m}\right)$, is approximately one monolayer $s \approx s_{m}$. This will give a very conservative estimate of the stability margin since, for example, the beam screen would ordinarily be designed to reach steady state with only a few per cent of a monolayer adsorbed. Second, we use eqn. 17 to evaluate the stability matrix for a finite length cold beam tube which has reached quasi steady state and is pumped from the ends without a beam screen present. Since the stability condition eqn. 17 is the same as for a warm beam tube, with the appropriate change in molecular velocity and the photodesorption gas load replaced by ordinary thermal outgassing, we also evaluate this case for room temperature beam tubes. The warm case is applicable to the long straight room temperature vacuum sections in the eight insertion regions of LHC (total beam tube length $\sim 3.2 \mathrm{~km}$ ) and to the detector beam tubes. Finally we use eqn. 20 to evaluate the stability matrix for a beam screen which is the most important case.

To avoid repetition we summarize here the additional data needed for numerical estimates of stability margins. We assume: $I=0.53 \mathrm{~A}, \sigma_{p i}\left(\mathrm{H}_{2}: \mathrm{CO}_{\mathrm{CO}}: \mathrm{CH}_{4}\right)=(2: 11: 17: 9) \times 10^{-19} \mathrm{~cm}^{2}$. Ion desorption coefficients of tightly bound molecules are taken, when available, from ref. 4 for a clean but otherwise unbaked and unconditioned metal surface with $\mathrm{H}_{2}{ }^{+}$and $\mathrm{CO}^{+}$energy $200 \mathrm{eV}$ in 
the arcs and room temperature straight sections and $3 \mathrm{keV}$ in the room temperature detector beam tube at the IP's. Estimation of the ion impact energy including the effect of bunching is treated in Appendix II. The specific values used at $200 \mathrm{eV}$ are: $\eta_{i}\left(\mathrm{H}_{2}{ }^{+}, \mathrm{H}_{2}\right)=0.50, \eta_{i}\left(\mathrm{H}_{2}{ }^{+}, \mathrm{CO}\right)=0.05$, $\eta_{i}\left(\mathrm{H}_{2}{ }^{+}, \mathrm{CO}_{2}\right)=0.025, \eta_{i}\left(\mathrm{H}_{2}{ }^{+}, \mathrm{CH}_{4}\right)=0.063, \eta_{i}\left(\mathrm{CO}^{+}, \mathrm{H}_{2}\right)=3.25, \eta_{i}\left(\mathrm{CO}^{+}, \mathrm{CO}\right)=2.0$, $\eta_{i}\left(\mathrm{CO}^{+}, \mathrm{CO}_{2}\right)=0.75, \eta_{i}\left(\mathrm{CO}^{+}, \mathrm{CH}_{4}\right)=0.25$, and at $3 \mathrm{keV}: \eta_{i}\left(\mathrm{H}_{2}{ }^{+}, \mathrm{H}_{2}\right)=1.7, \eta_{i}\left(\mathrm{H}_{2}{ }^{+}, \mathrm{CO}\right)=$ $0.22, \eta_{i}\left(\mathrm{H}_{2}{ }^{+}, \mathrm{CO}_{2}\right)=0.12, \eta_{i}\left(\mathrm{H}_{2}{ }^{+}, \mathrm{CH}_{4}\right)=0.23, \eta_{i}\left(\mathrm{CO}^{+}, \mathrm{H}_{2}\right)=11 ., \eta_{i}\left(\mathrm{CO}^{+}, \mathrm{CO}\right)=8.4$, $\eta_{i}\left(\mathrm{CO}^{+}, \mathrm{CO}_{2}\right)=3.7, \eta_{i}\left(\mathrm{CO}^{+}, \mathrm{CH}_{4}\right)=0.9$.

The appropriate molecular velocity in the pumping speeds appearing in eqns. 10,17 and 20 is the mean velocity of the molecules that have been desorbed by ion impact, whereas in the treatment given in Sec. I we have not kept track of the velocity of ion desorbed molecules compared to the non density dependent sources of gas in the numerators which initiate ion desorption. This is more or less intuitively expected, but will be demonstrated in Appendix I. However, since there is no experimental measurement of the velocity of molecules desorbed by ion impact, we will make the conservative choice of assuming it corresponds to the beam tube temperature.

\section{A. Infinitely long cold beam tube approximation}

As previously remarked the infinitely long cold beam tube approximation is interesting for the arcs of LHC as an initial transient before reaching the quasi steady state beam screen solution. It is also interesting for regions where there may be a cold beam tube and no significant photon flux and therefore no beam screen. The stability matrix we wish to evaluate is given by eqn. 10 . We will evaluate stability margins for: (a) $s\left(\mathrm{H}_{2}\right) / s_{m}\left(\mathrm{H}_{2}\right)=.02, s(\mathrm{CO}) / s_{m}(\mathrm{CO})=.06$, representative of the maximum surface density reached when a beam screen is present, and (b) a relatively thick layer $s\left(\mathrm{H}_{2}\right) / s_{m}\left(\mathrm{H}_{2}\right)=1, s(\mathrm{CO}) / s_{m}(\mathrm{CO})=3$, which in the presence of photodesorption is representative of the maximum surface densities that could be tolerated in most conceivable situations. Case (a) should also be adequate for the cold beam tube, no photon flux, no beam screen situation. There is some uncertainty about what to take for $s(\mathrm{CO}) / s_{m}(\mathrm{CO})$ since $\eta^{\prime} O$ hasn't been measured for $\mathrm{CO}$. If 
we assume $\eta^{\prime}$ is the same for $\mathrm{CO}$ and $\mathrm{H}_{2}$ at the same surface density, then $s(\mathrm{CO}) / s_{m}(\mathrm{CO}) \approx$ $3 s\left(\mathrm{H}_{2}\right) / s_{m}\left(\mathrm{H}_{2}\right)$, taking account of differences in monolayer normalizations, sticking coefficients and the magnitudes of photodesorption coefficients of tightly bound molecules. Finally, to give an idea how stable this situation is we will evaluate the stability matrix desorption coefficients for a third case (c) that has reached saturated ion desorption coefficients with $s\left(\mathrm{H}_{2}\right) / s_{m}\left(\mathrm{H}_{2}\right)=$ $\left.s(\mathrm{CO}) / s_{m} \mathrm{CO}\right) \sim 3$. The results for the three mentioned cases are given in Table I (a), (b) and (c) below.

In addition to the parameters given in the introduction to this section we give here the additional necessary information for the calculations in Table I. We have taken from ref. $4 \eta_{i}^{\prime}\left(H_{2}^{+}, H_{2}\right)=$ $1500\left(s\left(H_{2}\right) / s_{m}\left(H_{2}\right)\right)$ for $500 \mathrm{eV} \mathrm{H}_{2}{ }^{+}$, and $\eta_{i}^{\prime}\left(\mathrm{CO}^{+}, \mathrm{H}_{2}\right)=3000\left(s\left(\mathrm{H}_{2}\right) / s_{m}\left(\mathrm{H}_{2}\right)\right)$ for $500 \mathrm{eV} \mathrm{CO}{ }^{+}$, the minimum measurement energy in ref. 4 and about twice the magnitude of the time average potential expected in LHC. The value for $\eta_{i}^{\prime}\left(\mathrm{CO}^{+}, \mathrm{H}_{2}\right)$ has actually been inferred from measurements of $\mathrm{N}_{2}{ }^{+}$incident on physisorbed $\mathrm{H}_{2}$. For surface coverage less than a few monolayers and for the same ion energy $\mathrm{N}_{2}{ }^{+}$desorbs approximately twice as many $\mathrm{H}_{2}$ molecules as $\mathrm{H}_{2}{ }^{+}$. For the $\mathrm{H}_{2}$ monolayer normalization we have taken $s_{m}\left(H_{2}\right)=3 \times 10^{15} / \mathrm{cm}^{2}$. Direct measurements of $\eta_{i}^{\prime}\left(\mathrm{H}_{2}^{+}, \mathrm{CO}\right)$ and $\eta_{i}^{\prime}\left(\mathrm{CO}^{+}, \mathrm{CO}\right)$ do not seem to be available but we can make some reasonable estimates. Data were taken in ref. 4 for $5 \mathrm{keV} \mathrm{N}{ }_{2}+$ incident on condensed $N_{2}$ and it was found that the desorption yield was decreased a factor of $\sim 5$ compared to $5 \mathrm{keV} \mathrm{H}_{2}{ }^{+}$ on $\mathrm{H}_{2}$. We will assume the same situation holds for $500 \mathrm{eV} \mathrm{CO}{ }^{+}$on $\mathrm{CO}$ which have mass number 28 identical to $N_{2}$. We therefore obtain the estimate $\eta_{i}^{\prime}\left(\mathrm{CO}^{+}, \mathrm{CO}\right)=100\left(s(\mathrm{CO}) / \mathrm{s}_{m}(\mathrm{CO})\right)$ for $500 \mathrm{eV} \mathrm{CO}^{+}$and monolayer normalization $s_{m}(C O)=1 \times 10^{15} / \mathrm{cm}^{2}$. For $\eta_{i}^{\prime}\left(H_{2}^{+}, C O\right)$ we assume the factor of two decrease in desorption yield of $\mathrm{H}_{2}{ }^{+}$compared to $\mathrm{CO}^{+}$on $\mathrm{H}_{2}$ holds as well for desorption of $\mathrm{CO}$ and obtain for $500 \mathrm{eV} \mathrm{H} 2^{+}, \eta_{i}^{\prime}\left(\mathrm{H}_{2}^{+}, \mathrm{CO}\right)=50\left(s(\mathrm{CO}) / \mathrm{sm}_{m}(\mathrm{CO})\right)$. For the pumping speed of the beam tube wall we assume $\sigma_{w}\left(H_{2}\right)=\sigma_{w}(C O)=1, \bar{v}\left(H_{2}\right)=2.1 \times 10^{4}$ $\mathrm{cm} / \mathrm{s}, \quad \bar{v}(C O)=5.6 \times 10^{3} \mathrm{~cm} / \mathrm{s}$, so $\sigma_{w}\left(H_{2}\right) S_{w}\left(H_{2}\right)=761 / \mathrm{cm}-\mathrm{s}$ and $\sigma_{w}(C O) S_{w}(C O)=$ $20 \mathrm{l} / \mathrm{cm}-\mathrm{s}$. 
Looking first at the results in Table I(a) we see that the matrix elements of $R$ and the first order closed loop gains for $\mathrm{H}_{2}$ and $\mathrm{CO}$ are of order $10^{-3}$ while the second order coupled loop gain $G\left(H_{2}, C O\right)$ is of order $3 \times 10^{-7}$. This case is evidently quite stable, $\operatorname{det}(1-R)=0.998$, hardly different than unity. In this situation with $\operatorname{det}(I-R)$ dominated by the diagonal elements a useful estimate of the stability margin is the inverse of the largest diagonal element, $1 / R\left(\mathrm{CO}^{+}, \mathrm{CO}\right)=667$. Other gases, $\mathrm{CO}_{2}$ and $\mathrm{CH}_{4}$ have not been included but there is no reason to expect their matrix elements would not also be $\sim 10^{-3}$ and the stability margin would essentially be unchanged. The stabilizing influence of the pumping speed of the cold beam tube is so large that even when the accumulated $\mathrm{H}_{2}$ has reached the region of rapid rise in isotherm density at $s / s_{m}=1$ (Table I(b)) and saturated ion desorption coefficients (Table I(c)) the vacuum remains stable, although with reduced safety margin equal to 18 . The off diagonal element $\left.\mathrm{R}_{(\mathrm{CO}}{ }^{+} \mathrm{H}_{2}\right)$ is the largest matrix element in Table I but its magnitude is diminished in importance by the smallness of $\left.\mathrm{R}_{\left(\mathrm{H}_{2}\right.}{ }^{+} \mathrm{CO}\right)$ so $\operatorname{det}(I-R)$ is dominated by the diagonal elements. Even in the most extreme case of Table I(c) the second order closed loop gain is only $2.2 \times 10^{-3}$.

\section{B. Finite length beam tube with the surface in quasi equilibrium and pumped from the ends}

In Table II we have evaluated the stability matrix eqn. 17 for three cases: (a) a $L=5 \mathrm{~m}$ long $4.2 \mathrm{~K}$ beam tube with radius $r_{w}=2.3 \mathrm{~cm}$, (b) a $L=10 \mathrm{~m}$ long room temperature beam tube with radius $r_{w}=5.0 \mathrm{~cm}$ and (c) a $L=3.6 \mathrm{~m}$ long room temperature beam tube with radius $r_{w}=$ $4.0 \mathrm{~cm}$. The first case is mainly academic but corresponds according to Fig. 3 to the longest cold beam tube exposed to photons that one could conceivably pump from the ends without a beam screen and also the shortest cold component length in the LHC. The second case is important for room temperature straight sections and we have increased to beam tube radius to $5 \mathrm{~cm}$ since it is unconstrained by a superconducting magnet aperture and greatly facilities pumping from the ends. Finally the third case is applicable to the straight cylindrical section of beam tube at the center of 
one of the pp detectors of LHC. For Tables IIa and IIb we use ion desorption coefficients for 200 $\mathrm{eV}$ ions and for Table IIc, $3 \mathrm{keV}$ ions. From Table II(a) we see that the cold $L=5 \mathrm{~m}$ beam tube is predicted to be unstable with loop gain $G\left(\mathrm{CO}^{+}, \mathrm{CO}\right)=1.3>1$. This together with the marginal performance in Fig. 3 implies that a beam screen is necessary for essentially any practical case one can imagine for LHC where a cold beam tube is exposed to the design photon intensity $10^{17}$ photons $/ \mathrm{m} / \mathrm{sec}$. The room temperature case in Table II(b) is comfortably stable with $\operatorname{det}(1-R)=0.94$, close to unity, and stability margin $1 / R\left(\mathrm{CO}^{+}, \mathrm{CO}\right)=16.7$. Similarly the center section of the detector beam tube is stable with $\operatorname{det}(1-R)=0.87$ and stability margin $1 / R\left(\mathrm{CO}^{+}, \mathrm{CO}\right)=7.7$. The stability margin of last case will be reduced somewhat when the cylindrical cones between the central beam tube and nearest pumps are added, however the size of these connecting cones is to be specified so the conductance from the IP to the pumps is dominated by the central beam tube section.

\section{Beam tube with a beam screen}

The stability matrix eqn. 20 for a beam screen with $1 \%$ effective transparency, radius $r_{w}=$ $2.3 \mathrm{~cm}$ and other parameters relevant to LHC is given in Table III. Det $(1-R)=0.96$ is very close to unity so this case is stable. The second order closed loop gain $G\left(\mathrm{H}_{2}, \mathrm{CO}\right)=2.5 \times 10^{-} 6 \ll<$ is a negligible contribution to $\operatorname{det}(I-R)$ compared to the diagonal first order loop gains. The safety margin is therefore again the inverse of the largest diagonal element, $1 / R\left(C O^{+}, C O\right)=28$. The safety margin for $\mathrm{H}_{2}$ is approximately eighty times larger than for $\mathrm{CO}$. Since it is unlikely that a second order loop would become unstable before a first order loop, the most important matrix element missing from Table III is $\mathrm{R}\left(\mathrm{CO}_{2}{ }^{+}, \mathrm{CO}_{2}\right)$. We can estimate its importance from the magnitude of $\mathrm{R}\left(\mathrm{CO}^{+}, \mathrm{CO}_{2}\right)$ which is less than half of $\mathrm{R}\left(\mathrm{CO}^{+}, \mathrm{CO}\right)$. While $\mathrm{CO}_{2}{ }^{+}$may be somewhat more efficient desorbing $\mathrm{CO}_{2}$ than $\mathrm{CO}^{+}$, the difference should not be large so it is reasonable to expect $R\left(\mathrm{CO}_{2}{ }^{+}, \mathrm{CO}_{2}\right)$ is no larger than $R\left(\mathrm{CO}^{+}, \mathrm{CO}\right)$. In this case $\operatorname{det}(1-R)$ is reduced slightly to 0.929 but the safety margin is unchanged from Table III. 


\section{SUMMARY}

In this paper we have presented an analysis of ion desorption stability in the beam tube of superconducting proton storage rings. Emphasis has been placed on situations where significant fluxes of photodesorbing synchrotron radiation are encountered in a cold cryosorbing beam tube, as in the arcs of the LHC under construction at CERN. The primary new feature compared to previous analyses is the presence of physisorbed gas on the beam tube which, depending on the surface concentration, can have extremely high ion desorption coefficients. A generalized sojourn time of physisorbed molecules was defined to include thermal desorption, photodesorption and ion desorption. The ion desorption rate is density dependent and divergent at the onset of instability causing the sojourn time to approach zero. Experimental data were used to evaluate the $\mathrm{H}_{2}$ sojourn time for the conditions expected in the LHC. This situation was found to be stable even for a relatively thick layer of physisorbed $\mathrm{H}_{2}$; for surface densities less(greater) than a monolayer the sojourn time was found to be dominated by photodesorption(thermal desorption). When photodesorbing radiation is present it is known that the surface density of physisorbed $\mathrm{H}_{2}$ must be held to a few per cent of a monolayer, for example by employing a beam screen, in order to hold beam gas scattering to a tolerable level. For surface densities less than about $80 \%$ of a monolayer the sojourn time was found to be dominated by photodesorption, independent of surface density and equal to approximately $100 \mathrm{sec}$. At the level of a few per cent of a monolayer encountered with a beam screen the mean desorption time of a cryosorbed molecule by ion impact was estimated to exceed $5 \times 10^{4}$ sec.

The paper next considered the inclusion of gases heavier than hydrogen $\left(\mathrm{CO}, \mathrm{CO}_{2}\right.$ and $\left.\mathrm{CH}_{4}\right)$, the development of stability criteria and the evaluation of stability margins. A margin of stability was defined to be the factor by which the product of proton beam current and ion desorption coefficient would have to increase to cause an instability. Three interesting cases were treated: (1) a simple cryosorbing beam tube, (2) a cryosorbing beam tube pumped at the ends and (3) a 
cryosorbing beam screen. For all three cases ion desorption of a single component led to a simple inequality that must be satisfied for stability. Physically ion desorption is a regenerative feedback process and the inequality required that the net gain in production of gas molecules by ion desorption be less than unity. For the first case the surface concentration of physisorbed molecules continually increases due to adsorption of the previously tightly bound molecules that are freshly desorbed by photons. In this case the stability criterion contained the sum of ion desorption coefficients of the physisorbed and the tightly bound molecules. The stability criterion requires that the ion desorption rate per gas molecule must be less than the cryosorption pumping speed onto the entire beam tube surface. For the second and third cases the surface concentration reaches a quasi steady state and the generation rate of fresh molecules by photodesorption is balanced by pumping out the ends of the tube or through the perforations in the beam screen. In these cases the stability criterion did not contain the ion desorption coefficient of physisorbed molecules, which even for small surface concentrations of a few per cent exceeds the coefficient for ion desorption of tightly bound molecules by one to two orders of magnitude. On the other side of the inequality there was a compensating decrease in pumping speed from the pumping speed of the entire beam tube surface to the conductance out the ends of the tube or through the perforations covering a few per cent of the beam screen surface. There would be no gain in stability margin if the temperature of the beam screen were increased to avoid physisorption so it appears that the beam screen temperature can be chosen mainly from the viewpoint of engineering convenience and requirements. In each of the three cases generalization from a single gas component to a gas mixture led to replacing the simple inequality by a condition that a determinant be greater than zero. The elements of the determinant were shown to be a natural extension of the previous inequalities which now appear on the diagonal for each component. The off diagonal terms are due to ion desorption coupling of the molecular species. Because the gain of the off diagonal closed feedback loops is second or higher order in the ion desorption coefficients whereas the diagonal loops are first order, the stability determinants tend to be dominated by the diagonal terms in practical situations. Then the inverse of the largest first order closed loop gain was claimed to be a useful estimate of the stability margin. 
Generally there was only enough experimental information to evaluate the complete stability matrix for $\mathrm{H}_{2}$ and $\mathrm{CO}$. However it was argued, for example on the basis of the magnitude of the $\mathrm{CO}^{+}$, $\mathrm{CO}_{2}$ ) ion desorption coefficient, that this was enough to be reasonably confident of stability predictions considering $\mathrm{H}_{2}$ and $\mathrm{CO}$ only.

The ion desorption stability matrices were evaluated numerically for the anticipated conditions of the LHC. In all cases the ion desorption coefficients were assumed to be characteristic of solvent cleaned but otherwise untreated metal surfaces. The ion desorption energy was taken to be $200 \mathrm{eV}$ for the ions in the arcs and room temperature straight sections in the insertion regions. For the room temperature detector beam tube the ion energy at the IP was calculated to be $3.2 \mathrm{keV}$ for $\mathrm{CO}^{+}$ and $12 \mathrm{keV}$ for $\mathrm{H}_{2}{ }^{+}$, taking account of beam bunching. All of the cases examined were predicted to be stable with large stability margins except for one. The safety margin for a beam screen with $1 \%$ transparency was found to be 28 . A simple cryosorbing beam tube with a few per cent of a monolayer had a stability margin of 667 . This case is applicable to a cryosorbing beam screen before the surface reaches a quasi steady state and to a cold beam tube without a beam screen and not exposed to significant photodesorbing radiation or other sources of physisorbed gas. The safety margin was also evaluated for two room temperature cases in LHC, since the derived formulas apply there as well, and found to be 16.7 for an insertion region straight section and 7.7 for the beam tube at the center of a pp detector. The only case found to be unstable was a cryosorbing beam tube with the surface in quasi equilibrium and pumped from the ends. In that case the stability margin for a $5 \mathrm{~m}$ long tube with radius $2.3 \mathrm{~cm}$ was found to be less than one. Instability occurred in this case because of the very low conductance of the beam tube evaluated at 4.2 K. This case was evaluated for completeness and is not expected to be encountered in the LHC since a beam screen would ordinarily be in place in a region that would accumulate sufficient surface density to reach quasi equilibrium. 


\section{ACKNOWLEDGMENT}

The author gratefully acknowledges helpful conversations with Roger Calder, Miguel Furman, Oswald Grobner and Alastair Mathewson during the course of the work described in this paper.

\section{APPENDIX I: GENERALIZATION TO MULTIPLE VELOCITY COMPONENT GAS MIXTURES}

In sections III and IV we remarked that we have written equations with only a single velocity component characterizing each molecular species. In principle the mean velocity of molecules due

to photodesorption, thermal desorption and ion desorption can all be different. The single velocity appearing in the pumping speeds, conductances and diffusion coefficients in our equations can then be identified with the density weighted average of the component velocities.[2] Here we want to keep track of the velocity of molecules desorbed by ion impact. Let subscript "1" designate photodesorbed tightly bound molecules, "2" photodesorbed physisorbed molecules, "3" thermally desorbed molecules and "4" ion desorbed molecules. For an example we treat the infinitely long beam tube case of Sec. IIA. The density equations for each component are:

$$
\begin{aligned}
& V \frac{\partial n_{1}}{\partial t}=\eta_{1} \dot{\Gamma}-\sigma_{w 1} S_{w 1} n_{1} \\
& V \frac{\partial n_{2}}{\partial t}=\eta^{\prime} \dot{\Gamma}-\sigma_{w 2} S_{w 2} n_{2} \\
& V \frac{\partial n_{3}}{\partial t}=\frac{A_{w} s}{\tau_{w}^{t}}-\sigma_{w 3} S_{w 3} n_{3} \\
& V \frac{\partial n_{4}}{\partial t}=\left(\eta_{i}+\eta_{i}^{\prime}\right)\left(\frac{I \sigma_{p i}}{e}\right)\left(n_{1}+n_{2}+n_{3}+n_{4}\right)-\sigma_{w 4} S_{w 4} n_{4}
\end{aligned} .
$$

The quasi steady state solution to eqns. A1 is: 


$$
\begin{aligned}
n & =\frac{\sigma_{w 4} S_{w 4}}{\sigma_{w 4} S_{w 4}-\eta_{i} \frac{I \sigma_{p i}}{e}-\eta_{i}^{\prime} \frac{I \sigma_{p i}}{e}} \times\left(\frac{\eta_{1} \dot{\Gamma}}{\sigma_{w 1} S_{w 1}}+\frac{\eta^{\prime} \dot{\Gamma}}{\sigma_{w 2} S_{w 2}}+\frac{A_{w s} s}{\tau_{w}^{t}}\right) \\
& =\frac{1}{1-G} \times\left(\frac{\eta_{1} \dot{\Gamma}}{\sigma_{w 1} S_{w 1}}+\frac{\eta^{\prime} \dot{\Gamma}}{\sigma_{w 2} S_{w 2}}+\frac{A_{w} s}{\tau_{w}^{t}}\right)
\end{aligned} .
$$

As before ion desorption is equivalent to a multiplier of the density in the absence of ion desorption. Now however the multiplier contains the parameters specific to ion desorption and in particular the expression for the gain given by eqn. 9 appears with the molecular velocity explicitly identified as the velocity of molecules desorbed by ion impact.

\section{APPENDIX II: ESTIMATION OF THE IMPACT ENERGY OF IONS HITTING THE BEAM TUBE TAKING ACCOUNT OF PROTON BEAM BUNCHING}

In the main text of the paper we have used ion desorption coefficients corresponding to the maximum ion energies expected in LHC due to acceleration in the space charge field of the beam. In this Appendix we give the estimates of these energies that include taking account of beam bunching. The axial bunch profile is assumed uniform with length $c \tau$ and distance between bunches $c T$. The peak and time average beam currents are then related by $\hat{I} \tau=I T$. For LHC $\tau=$ $0.25 \mathrm{~ns}$ and $T=25 \mathrm{~ns}$. Due to quadrupole focusing the area and the shape of the transverse cross section of the beam depend on position in the accelerator lattice and this can influence the magnitude of the electric field strength accelerating ionized gas molecules. Consider a transverse beam density distribution that is uniform inside an elliptical boundary. In that case it can be shown that: (1) the maximum magnitude of electric field strength is on the elliptical boundary, (2) the magnitude is constant on the boundary and (3), assuming constant cross section area, the maximum field strength is for ellipticity equal to one, or a circular boundary. ${ }^{11}$ In a FODO lattice the cross section area of the beam is not constant however in the standard LHC cell it varies only $\pm 2.5 \%$. The ellipticity $\varepsilon$ varies from $\sim 2.4$ at a quadrupole 1.0 at the midplane of a half cell 
where the cross section is circular. For constant cross section area the magnitude of field strength on the boundary varies as $\varepsilon /(1+\varepsilon)^{2}$ and increases $\sim 20 \%$ passing from a quadrupole to half cell midplane. It is reasonable therefore to estimate field strengths and ion energies for a circular cross section beam with lattice betatron function and beam size equal to their values at a half cell midplane. We will do this for uniform and Gaussian radial beam profiles.

The rms beam size in one transverse dimension is given by $\sigma=\sqrt{\beta \varepsilon_{n} / \gamma}$ where $\varepsilon_{n}=$ $3.75 \times 10^{-6} \mathrm{~m}-\mathrm{rad}$ is the normalized emittance and $\beta$ is the lattice betatron function $(=80.2 \mathrm{~m}$ at the center of an LHC half cell). For a $7 \mathrm{TeV}$ proton beam $\gamma=7460$ and we obtain $\sigma=0.20 \mathrm{~mm}$. The corresponding rms radius is $\sigma_{r}=(2)^{1 / 2} \sigma=0.28 \mathrm{~mm}$. This estimate of beam radius is adequate for the arcs and the room temperature straight sections of LHC. At the interaction points (IP's) where the two large pp detectors ATLAS and CMS are to be located, the beam is tightly focused for high luminosity and the beam size is much reduced; $\beta^{*}=0.5 \mathrm{~m}, \sigma_{r}{ }^{*}=22.5 \mu \mathrm{m}$. Ion energies will be calculated for these two estimates of rms radius.

For a uniform radial beam current profile the time average electric field is given by;

$$
\begin{aligned}
& E=\frac{I Z_{0}}{2 \pi} \frac{r}{a^{2}}, \quad r<a, \\
& E=\frac{I Z_{0}}{2 \pi} \frac{1}{r}, \quad r \geq a,
\end{aligned}
$$

and the time average electric potential between the beam axis $r=0$ and tube at $r=r_{w}$ is;

$$
\Phi=\frac{I Z_{0}}{2 \pi}\left(\ln \frac{r_{w}}{a}+0.5\right)
$$

The rms radius of a uniform distribution is $\sigma_{r}=a /(2)^{1 / 2}$. For $I=0.53 \mathrm{~A}, \sigma_{r}=0.28 \mathrm{~mm}, r_{w}=$ $23 \mathrm{~mm}$ in an arc cell, $\Phi=145 \mathrm{~V}$ and for $I=2 \times 0.53 \mathrm{~A}, \sigma_{r}=22.5 \mu \mathrm{m}, r_{w}=40 \mathrm{~mm}$ at an IP, $\Phi=$ $486 \mathrm{~V}$. Peak fields due to bunching are $T / \tau=100$ times higher. 
For a Gaussian profile the time average electric field is given by,

$$
E=\frac{I Z_{0}}{2 \pi} \frac{1-e^{-\frac{r^{2}}{\sigma_{r}^{2}}}}{r}
$$

Numerically integrating from the beam axis to the beam tube, the potential is $149 \mathrm{~V}$ for the arc cell case and $495 \mathrm{~V}$ for the IP case, not too different than for the uniform distribution given in the previous paragraph.

If the beam were unbunched then ions would arrive at the beam tube with kinetic energy equal to the difference in potential between the point of ionization and the beam tube. However if the beam is bunched and the ion inertia is such that the ions move significantly on the time scale of a single bunch passage then they can arrive at the wall with an energy exceeding the time average beam potential. The lighter the mass the more pronounced the effect; an upper bound is the peak potential $\hat{\Phi}=\frac{T}{\tau} \Phi$ during the passage of a single bunch. Practical cases require numerical integration. We will begin with the uniform profile and assume an ion is produced at the head of a bunch at radius $r=a$. The magnitude and duration of electric field strength and energy kick for the first bunch are then maximized. If we neglect ion motion during the passage of a bunch the radial position and velocity at the head of the $\mathrm{n}$ th bunch are;

$$
\begin{aligned}
& v_{n}=v_{n-1}+\frac{e}{m} \frac{\hat{I} \tau Z_{0}}{2 \pi} \frac{1}{x_{n-1}} . \\
& x_{n}=x_{n-1}+v_{n} T
\end{aligned}
$$

For the calculations done below Eqn. A6 is a good approximation for all but the first bunch. For the first bunch ion motion during the bunch passage can be significant and the equation of motion must then be integrated numerically with time steps chosen small enough not to influence the final 
result. The ion energy and radial position preceding each bunch passage are shown in Fig. 6 for $\mathrm{H}_{2}{ }^{+}$and $\mathrm{CO}^{+}$for the arc cell and IP cases of LHC discussed above. For the arc cell case Fig. 6a $\mathrm{H}_{2}{ }^{+}$reaches the beam tube with energy $176 \mathrm{eV}$ after the passage of the eighth bunch, for $\mathrm{CO}^{+}$the energy is $130 \mathrm{eV}$ and thirty seven bunches have passed. For comparison the time average beam potential from $r=a$ to $r=r_{w}$ is $128.6 \mathrm{~V}$. The mass of $\mathrm{CO}^{+}$is large enough that bunching has had only a small effect on the ion energy. For the lighter mass $\mathrm{H}_{2}{ }^{+}$the effect of bunching is more pronounced and has increased the ion energy at the beam tube by $\sim 40 \%$. The IP case with much smaller beam radius in Fig. $6 \mathrm{~b}$ is more dramatic; $\mathrm{H}_{2}{ }^{+}$reaches the beam tube with $12 \mathrm{keV}$ after passage of two bunches and $\mathrm{CO}^{+}$with $3.2 \mathrm{keV}$ after eleven bunches. In both cases the ions get essentially all their energy from the first bunch and then drift to large enough radius that the following bunches have little effect. Motion during the passage of the first bunch while not negligible is still small compared to $r_{w}$ so ions reach the wall with energy much less than the peak potential. At an IP the time average beam potential from $r=a$ to $r=r_{w}$ is $454 \mathrm{~V}$ and the peak potential is $45.4 \mathrm{kV}$.

For the Gaussian beam profile there is an additional factor $1-e^{-\frac{x_{n-1}^{2}}{\sigma^{2}}}$ multiplying the second term on the right of eqn. A6a and we have repeated the calculation of Fig. 6a. In this case the peak electric field strength is at $r=1.12 \sigma_{r}$ and this was taken as the ion initial position. $\mathrm{H}_{2}{ }^{+}$was calculated to reach the wall with $165 \mathrm{eV}$ and $\mathrm{CO}^{+}$with $134 \mathrm{eV}$, hardly different than the uniform profile.

The results of this Appendix have been used to specify the ion energy of the ion desorption coefficients. In all cases except the IP we have taken $200 \mathrm{eV}$. For the IP we take $3 \mathrm{keV}$, the maximum energy of the experimental ion desorption coefficient data. This corresponds closely to the energy estimated for $\mathrm{CO}^{+}$but is considerably less than the $12 \mathrm{keV}$ calculated for $\mathrm{H}_{2}{ }^{+}$. Experimentally the magnitude of ion desorption coefficients increases with energy but appear to roll off at $1 \mathrm{keV}$ and approach a nearly constant value by $3 \mathrm{keV}^{4}$ so we do not think a significant error is being made. 


\section{REFERENCES}

1W.C. Turner, Proc. of 1993 IEEE Part. Acc. Conf., Washington, D.C., p. 3833 (1993).

2 W.C. Turner, "Beam Tube Vacuum in Future Superconducting Proton Colliders", AIP Conf.

Proc. 326, Accelerator Physics at the Superconducting Super Collider, eds. Y. Yan, J. Naples and M. Syphers, p. 326 (1995).

${ }^{3}$ LHC, The Large Hadron Collider Accelerator Project, eds. Y. Baconnier, G. Brianti, Ph. Lebrun, A. Mathewson and R. Perin, CERN/AC/93-03 (1993).

${ }^{4}$ A. G. Mathewson, CERN-ISR-VA/76-5 (1976).

$5_{N}$. Hilleret and R. Calder, Proc. of 7th Intern. Vac. Congress and 3rd Intern. Conf. Solid Surfaces, p. 227, (Vienna, 1977).

6V. Anashin et. al., J. Vac. Sci. Tech., A12, 2917 (1994).

7P. Redhead et. al., The Physical Basis of Ultrahigh Vacuum, AIP (1993).

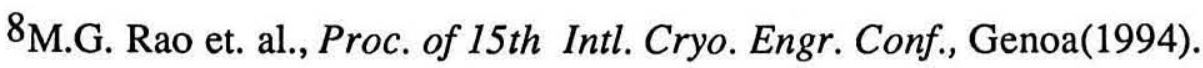

${ }^{9}$ R. Calder, Vacuum, 24, 437 (1974).

10N. Alinovsky et. al., Proc. of 1994 EPAC, London.

11M.A. Furman, Am. J. Phys., 62, 1134 (1994). 
Table I: Estimates of the ion desorption stability matrix $R\left(m k^{+}, m_{j}\right)$ for the infinitely long cold beam tube approximation.

(a). At transition to the beam screen solution; $s\left(H_{2}\right) / s_{m}=.02, s(C O) / s_{m}(C O)=.06$

$$
\mathrm{H}_{2}^{+} \mathrm{CO}^{+}
$$

\begin{tabular}{lll}
\hline $\mathrm{H}_{2}$ & $2.7 \times 10^{-4}$ & $3.0 \times 10^{-3}$ \\
$\mathrm{CO}$ & $1.0 \times 10^{-4}$ & $1.5 \times 10^{-3}$ \\
& $\operatorname{Det}(I-R)=0.998$, stable & \\
Stability margin $\sim 1 / R\left(C O^{+}, \mathrm{CO}\right)=667$ & \\
\hline
\end{tabular}

(b). At fifty times the surface density of physisorbed molecules as case (a); $s\left(\mathrm{H}_{2}\right) / s_{m}=1.0$, $\underline{\underline{s(C O)} / s_{m}(C O)=3.0 \text {. }}$

\begin{tabular}{lcc}
\hline \hline & $\mathrm{H}_{2}{ }^{+}$ & $\mathrm{CO}^{+}$ \\
\hline $\mathrm{H}_{2}$ & .013 & .144 \\
$\mathrm{CO}$ & $5.0 \times 10^{-3}$ & .055 \\
& Det $(I-R)=0.932$, stable & \\
& Stability margin $\sim 1 / R\left(C O^{+}, C O\right)=18.2$ & \\
\end{tabular}

(c). At saturation of ion desorption coefficients for physisorbed $\mathrm{H}_{2}$ and $\mathrm{CO} ; \mathrm{s}\left(\mathrm{H}_{2}\right) / s_{m}=3.0$, $s(C O) / s_{m}(C O)=3.0$

$$
\mathrm{H}_{2}^{+} \mathrm{CO}^{+}
$$

$\begin{array}{lcc}\mathrm{H}_{2} & .040 & 0.43 \\ \mathrm{CO} & 5.0 \times 10^{-3} & .055 \\ \operatorname{Det}(1-R)=0.905, \text { stable } & \\ \text { Stability } & \text { margin } ~ & 1 / R\left(H_{2}+H_{2}\right)=18.2\end{array}$

Stability margin $\sim 1 / R\left(H_{2}{ }^{+}, H_{2}\right)=18.2$ 
Table II: Estimates of the ion desorption stability matrix $R\left(m k^{+}, m_{j}\right)$ for finite length beam tubes with the surface in quasi equilibrium and pumped from the ends, no beam screen.

(a). The cold beam tube case, $L=5 \mathrm{~m}, r_{\mathcal{W}}=2.3 \mathrm{~cm}$

$\mathrm{H}_{2}^{+} \mathrm{CO}^{+}$

$\begin{array}{lcc}\mathrm{H}_{2} & .016 & 0.56 \\ \mathrm{CO} & 5.9 \times 10^{-3} & 1.3 \\ \mathrm{CO}_{2} & 3.7 \times 10^{-3} & 0.57 \\ \mathrm{CH}_{4} & 5.7 \times 10^{-3} & 0.13 \\ \operatorname{Det}(1-\mathrm{R})^{*}=-0.3<0, \mathrm{R}_{\left(\mathrm{CO}^{+}, \mathrm{CO}\right)}=1.3>1, \text { unstable }\end{array}$

(b). The warm (room temperature) beam tube case applicable to the straight sections of the LHC, $L=10 \mathrm{~m}, r_{w}=5 \mathrm{~cm}$

\begin{tabular}{lcc}
\hline \hline & $\mathrm{H}_{2}^{+}$ & $\mathrm{CO}^{+}$ \\
\hline $\mathrm{H}_{2}$ & $7.3 \times 10^{-4}$ & .026 \\
$\mathrm{CO}$ & $2.7 \times 10^{-4}$ & .06 \\
$\mathrm{CO}_{2}$ & $1.7 \times 10^{-4}$ & .026 \\
$\mathrm{CH} 4$ & $2.6 \times 10^{-4}$ & $5.7 \times 10^{-3}$ \\
& & \\
& Det $(1-\mathrm{R})^{*}=0.94$, stable & \\
Stability margin $1 / \mathrm{R}^{+}\left(\mathrm{CO}^{+}, \mathrm{CO}\right)=16.7$ & \\
& \\
\hline \hline
\end{tabular}


Table II cont'd

(c). Room temperature detector beam tube, $L=3.6 \mathrm{~m}, r_{w}=4 \mathrm{~cm}$

\begin{tabular}{lcc}
\hline \hline & $\mathrm{H}_{2}{ }^{+}$ & $\mathrm{CO}^{+}$ \\
\hline $\mathrm{H}_{2}$ & $1.25 \times 10^{-3}$ & $4.5 \times 10-2$ \\
$\mathrm{CO}$ & $6.1 \times 10^{-4}$ & .13 \\
$\mathrm{CO}_{2}$ & $4.2 \times 10^{-4}$ & .07 \\
$\mathrm{CH} 4$ & $4.8 \times 10^{-4}$ & .01 \\
& & \\
Det $(1-\mathrm{R})^{*}=0.869$, stable & \\
Stability margin $1 / R\left(\mathrm{CO}^{+}, \mathrm{CO}\right)=7.7$ & \\
\end{tabular}

*Det $(1-R)$ is only evaluated for $\mathrm{H}_{2}$ and $\mathrm{CO}$. 
Table III: Estimates of the ion desorption stability matrix $R\left(m k^{+}, m_{j}\right)$ for a cold beam tube with a $1 \%$ transparency beam screen

\begin{tabular}{llc}
\hline \hline & $\mathrm{H}_{2}{ }^{+}$ & $\mathrm{CO}^{+}$ \\
\hline $\mathrm{H}_{2}$ & $4.4 \times 10^{-4}$ & .016 \\
$\mathrm{CO}$ & $1.6 \times 10^{-4}$ & .036 \\
$\mathrm{CO} 2$ & $1.0 \times 10^{-4}$ & .016 \\
$\mathrm{CH} 4$ & $1.5 \times 10^{-4}$ & $3.4 \times 10^{-3}$ \\
& & \\
& & \\
Det $(I-R)^{*}=0.964$, stable & \\
Stability margin $=1 / R\left(C O^{+}, \mathrm{CO}\right)=28$. &
\end{tabular}

${ }^{*} \operatorname{Det}(I-R)$ is only evaluated for $\mathrm{H}_{2}$ and $\mathrm{CO}$. 


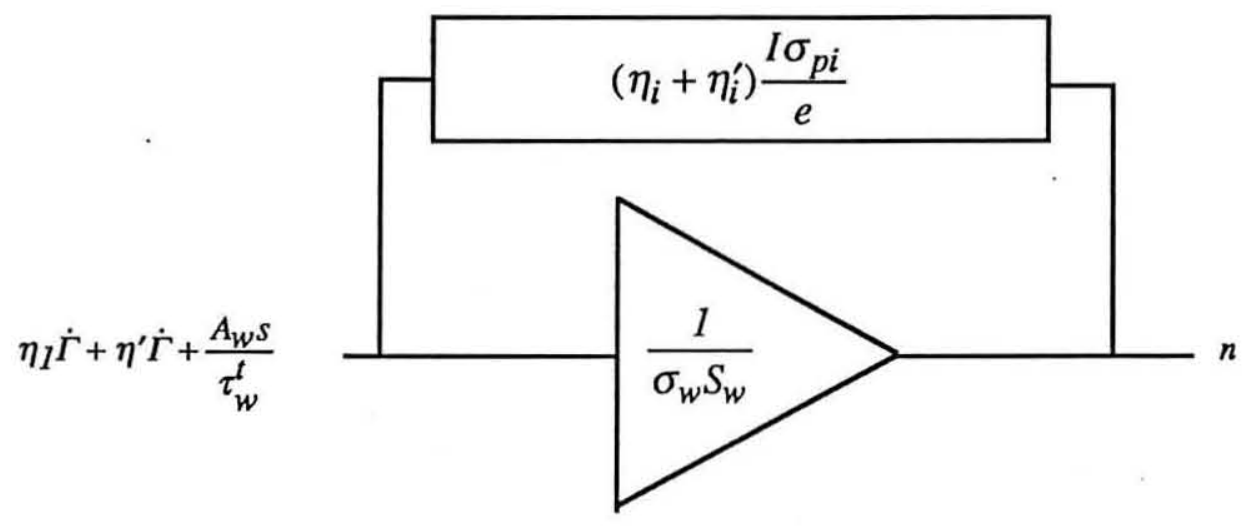

Fig. 1: Ion desorption feedback diagram for a single component system. 


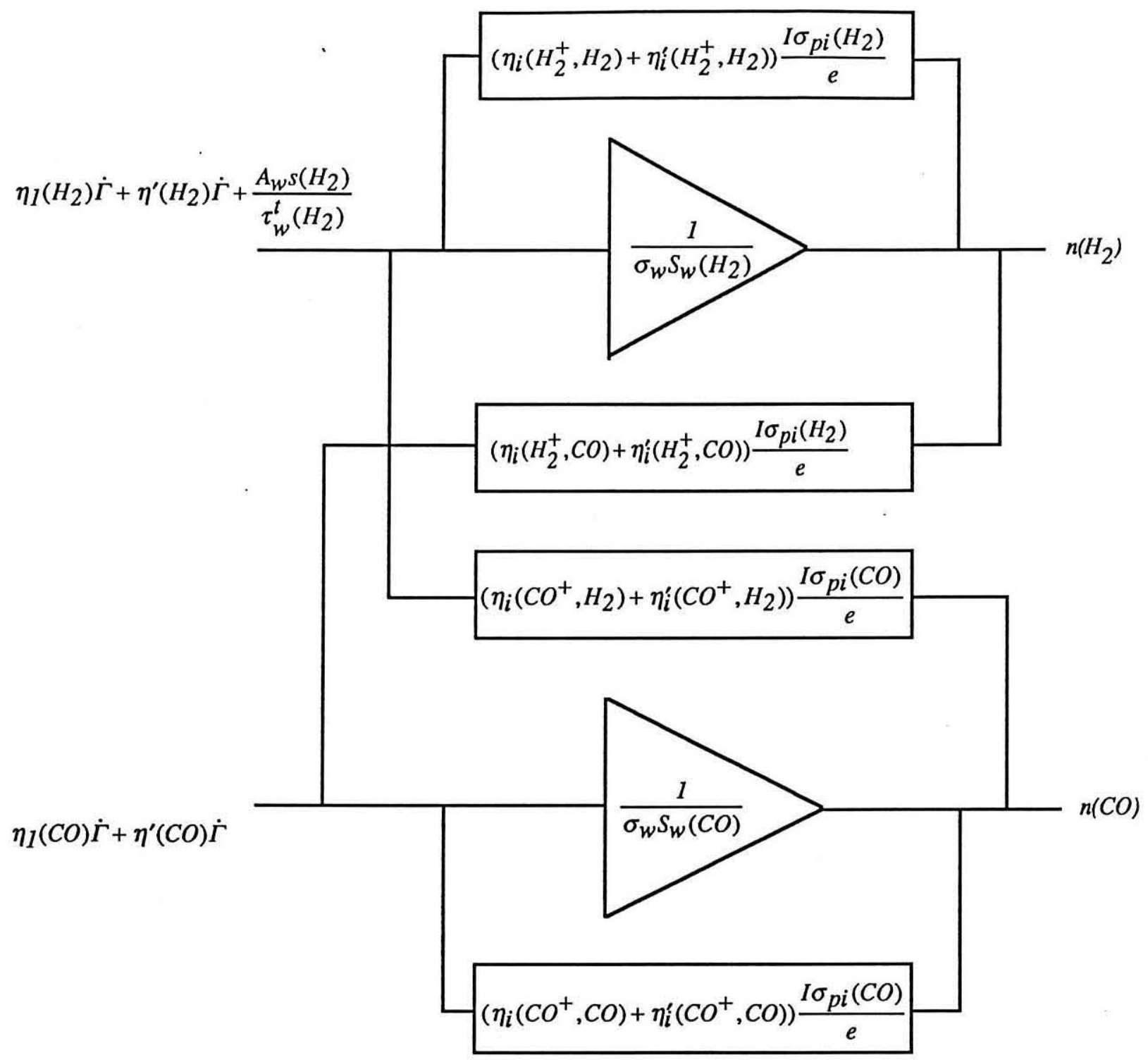

Fig. 2: Ion desorption feedback diagram for a two component $\mathrm{H}_{2}, \mathrm{CO}$ system, illustrating the second order nature of the loop which couples $\mathrm{H}_{2}$ and $\mathrm{CO}$. 


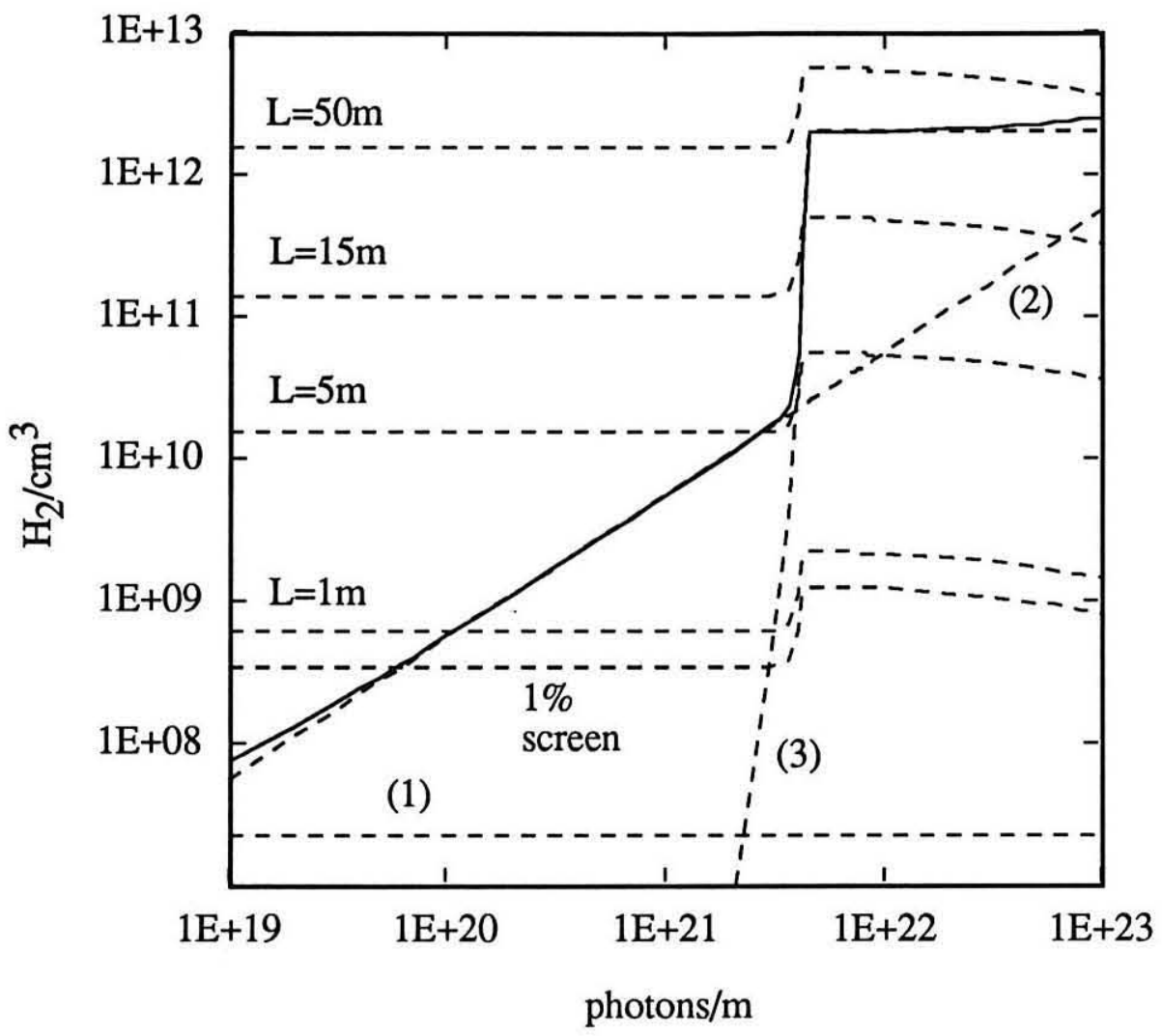

Fig. 3: $\mathrm{H}_{2}$ density versus photon flux for (a) an infinitely long beam tube (solid line), (b) for finite length beam tubes $L=1,5,15$ and $50 \mathrm{~m}$ and (c) for a $1 \%$ beam screen, all at $4.2 \mathrm{~K}$. Components of (a) are labeled (1) desorption of tightly bound $\mathrm{H}_{2}$, (2) desorption of physisorbed $\mathrm{H}_{2}$ and (3) thermal desorption. 


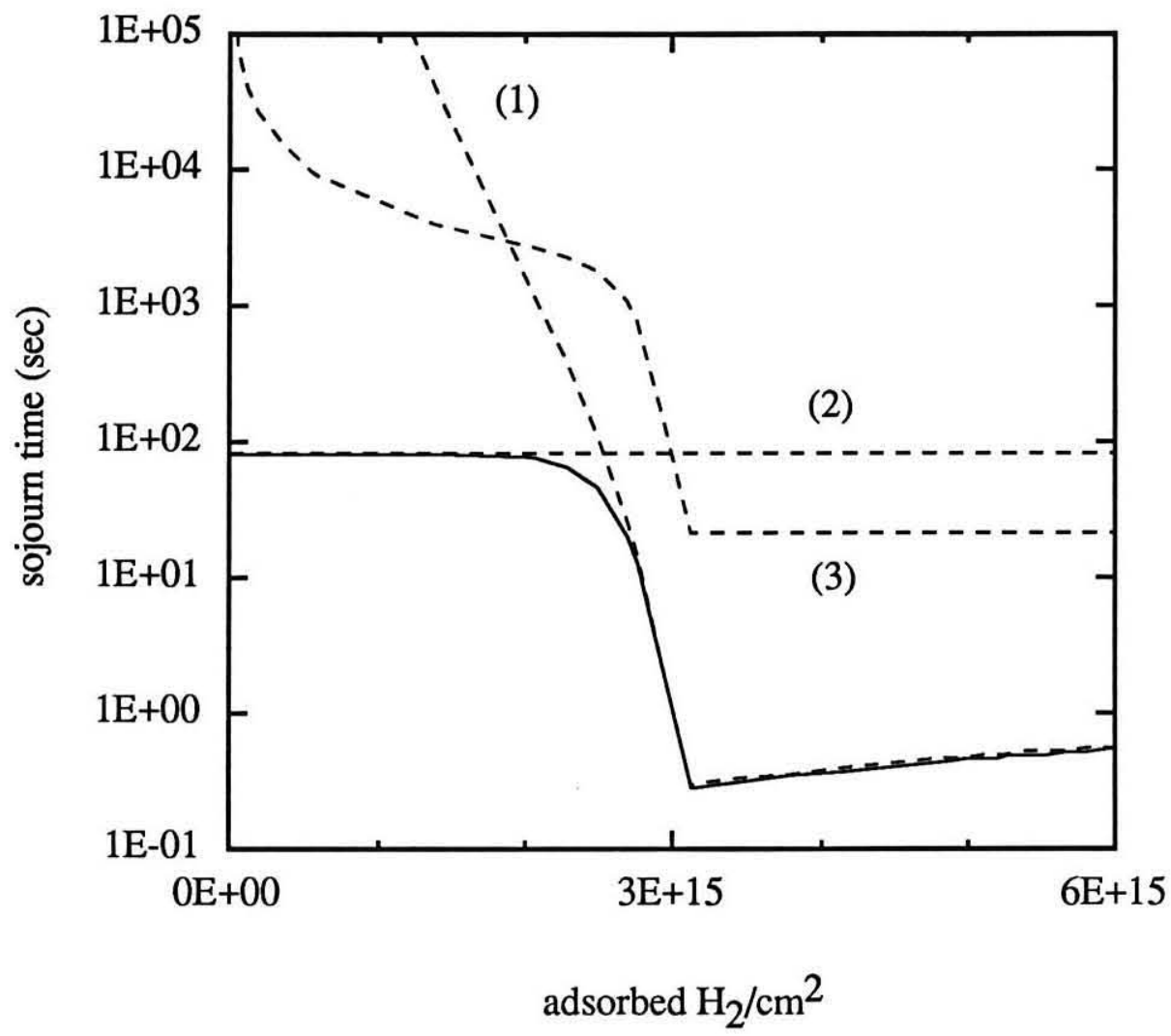

Fig.4: Effective $\mathrm{H}_{2}$ sojourn time due to (1) thermal, (2) photon and (3) ion desorption. 


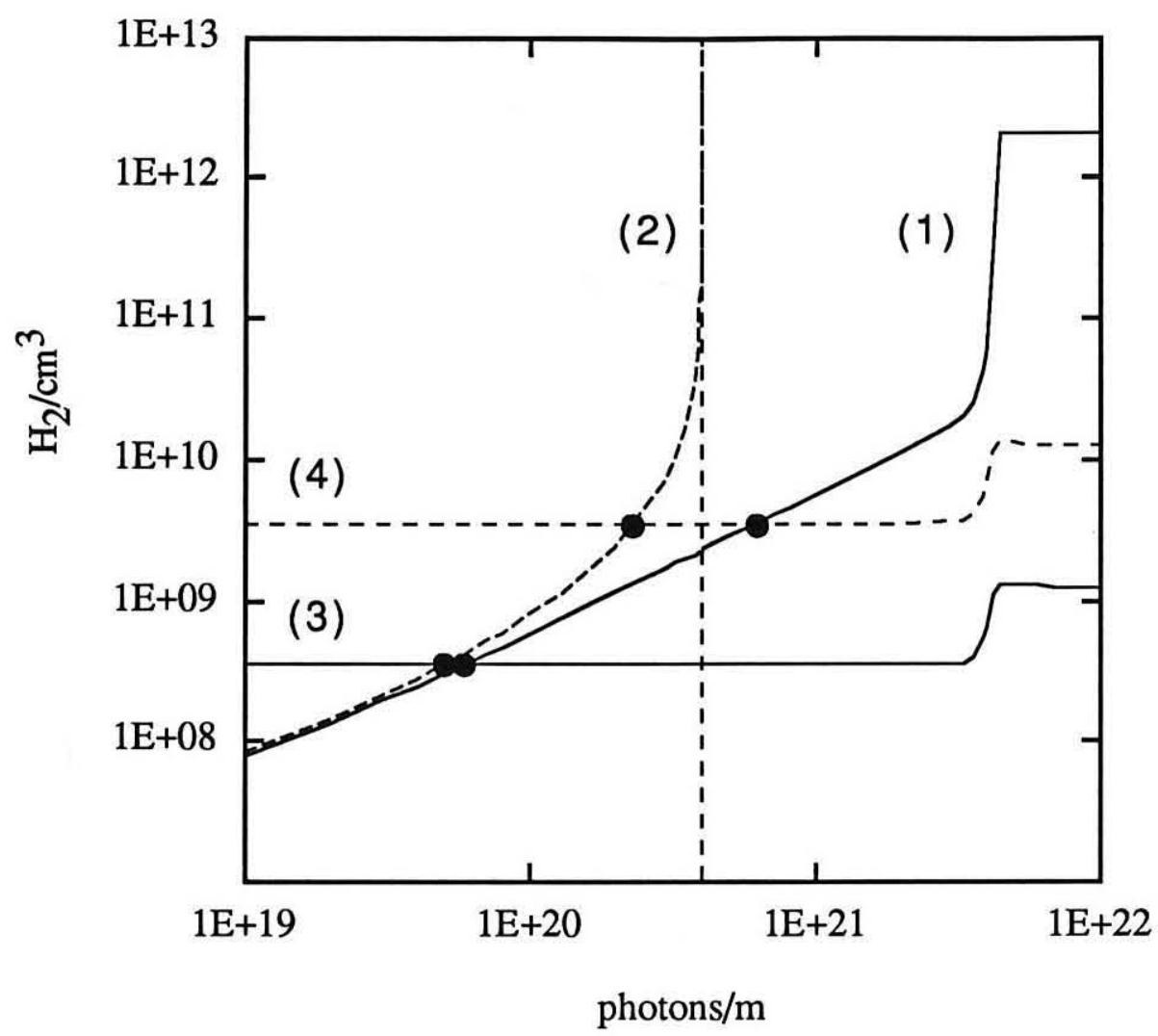

Fig. 5: $\mathrm{H}_{2}$ density versus photon flux illustrating the effects of ion desorption for hypothetical situations. Cases (1) and (2) are the infinite length cold beam tube solutions, cases (3) and (4) are 1\% transparency beam screen solutions. Ion desorption is negligible in (1) and (3). Case (2) becomes unstable at $\Gamma=4 \times 10^{20}$ photons $/ \mathrm{m}$, when the density of physisorbed $\mathrm{H}_{2}$ has reached approximately $10 \%$ of a monolayer. In case (4) the ion desorption denominator has increased the density one order of magnitude compared to (3). 


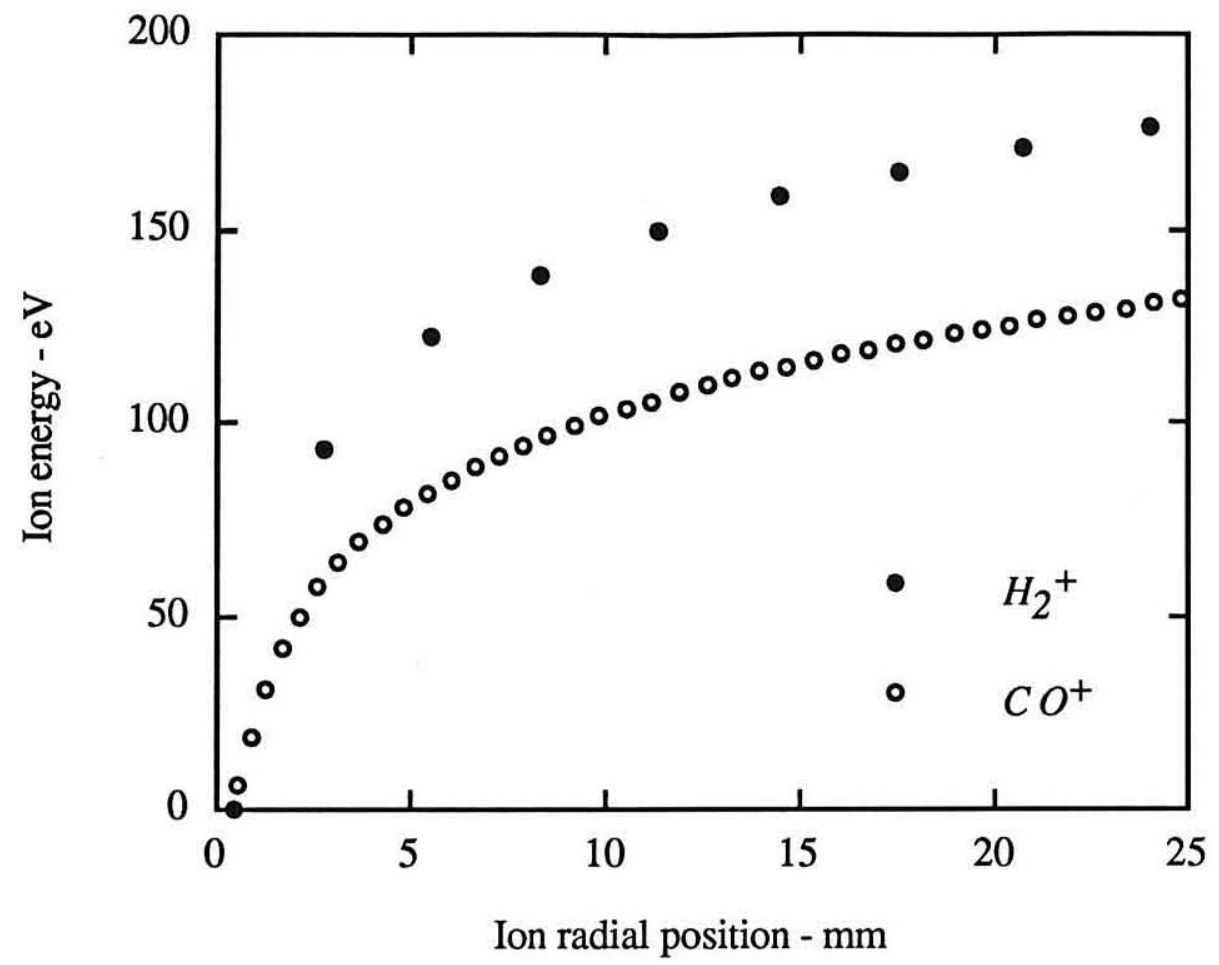

Fig. 6(a): Ion energy versus radial position at the head of successive proton bunches in the collider arcs. Results are shown for $\mathrm{H}_{2}{ }^{+}$and $\mathrm{CO}^{+}$and the uniform radial beam profile with rms radius $\sigma_{r}=$ $0.285 \mathrm{~mm}$ and beam tube radius $r_{w}=23 \mathrm{~mm}$. 


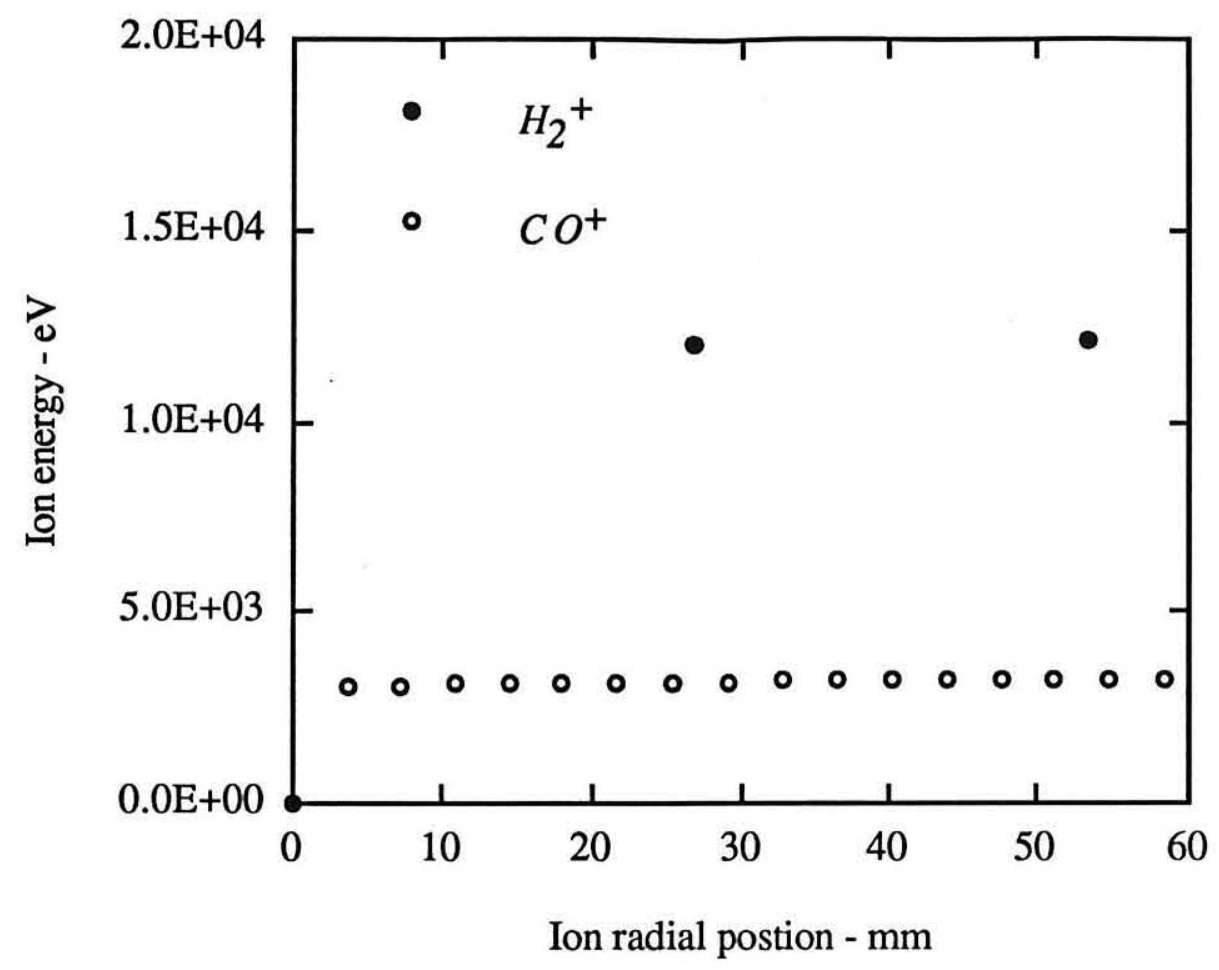

Fig. 6(b): Ion energy versus radial position at the head of successive proton bunches at a collider IP. Results are shown for $\mathrm{H}_{2}{ }^{+}$and $\mathrm{CO}^{+}$ and the uniform radial beam profile with rms radius $\sigma_{r}{ }^{*}=0.0225 \mathrm{~mm}$ and beam tube radius $r_{w}=40 \mathrm{~mm}$. 